\title{
Coupled Alternating Neural Networks for Solving Multi- Population High-Dimensional Mean-Field Games with Stochasticity
}

This paper was downloaded from TechRxiv (https://www.techrxiv.org).

\section{LICENSE}

CC BY 4.0

SUBMISSION DATE / POSTED DATE

$24-01-2022$ / 28-01-2022

\section{CITATION}

Wang, Guofang; Yao, Wang; Zhang, Xiao; Niu, Zijia (2022): Coupled Alternating Neural Networks for Solving Multi-Population High-Dimensional Mean-Field Games with Stochasticity. TechRxiv. Preprint. https://doi.org/10.36227/techrxiv.19009463.v1

$\mathrm{DOI}$ 


\title{
Coupled Alternating Neural Networks for Solving Multi-Population High-Dimensional Mean-Field Games with Stochasticity
}

\author{
Guofang Wang, Wang Yao, Xiao Zhang, and Zijia Niu
}

\begin{abstract}
Multi-population mean-field game is a critical subclass of mean-field games (MFGs). It is a theoretically feasible multi-agent model for simulating and analyzing the game between multiple heterogeneous populations of interacting massive agents. Due to the factors of game complexity, dimensionality disaster and disturbances should be taken into account simultaneously, how to solve the multi-population high-dimensional stochastic MFG problem is faced with great challenges. We present $\mathrm{CA}$ Net, a coupled alternating neural network approach for tractably solving multi-population high-dimensional MFGs in the stochastic case. First, we provide a universal modeling framework for large-scale heterogeneous multi-agent game systems, which is strictly expressed as a multi-population MFG problem. Next, we generalize the potential variational primal-dual structure that MFGs exhibit, then phrase the multi-population MFG problem as a convex-concave saddle-point problem. Last but not least, we design a generative adversarial network (GAN) with multiple generators and multiple discriminators-the solving network, which parameterizes the value functions and the density functions of multiple populations by two sets of neural networks, respectively. Moreover, numerical experiments demonstrate the feasibility and effectiveness of our approach.
\end{abstract}

Index Terms-Multi-population model, mean-field game (MFG), neural networks, high-dimensional solution space.

\section{INTRODUCTION}

$\mathbf{M}$ EAN-FIELD games (MFGs) [1]-[6] are a class of models that simulate and analyze large populations of interacting agents. The MFGs theory can be used to solve the communication and calculation difficulties caused by large-scale multi-agent system (MAS) scenarios, that is, the core is to transform the 1 -vs- $N$ game into a 1-vs-1 game form. In other words, the communication and computational complexities are no longer related to the agent number [7]. They are widely used in economics [8]-[11], finance [12][15], industrial engineering [16]-[18], crowd motion [19][22], swarm robotics [23], [24], epidemic modeling [25], [26], data science [27]-[29], and material dynamics [30].

Manuscript received XX, XX; revised XX, XX. (Corresponding author: Wang Yao and Xiao Zhang.)

G. Wang, X. Zhang and Z. Niu are with the School of Mathematical Sciences, Beihang University, Beijing 100191, China; Key Laboratory of Mathematics, Informatics and Behavioral Semantics, Ministry of Education, Beijing Advanced Innovation Center for Big Data and Brain Computing, Beihang University, Beijing 100191, China; Peng Cheng Laboratory, Shenzhen 518055, Guangdong, China e-mail: (xiao.zh@ buaa.edu.cn).

W. Yao was with the Institute of Artificial Intelligence, Beihang University, Beijing 100191, China; Key Laboratory of Mathematics, Informatics and Behavioral Semantics, Ministry of Education, Beijing Advanced Innovation Center for Big Data and Brain Computing, Beihang University, Beijing 100191, China e-mail: (yaowang @ buaa.edu.cn).
From the perspective of the implementation of MFGs theory, we will find that although MFGs theoretical research is relatively advanced and mature, its numerical solution technology is far from reaching the needs of actual scenarios [31]. Many researchers have made efforts on the numerical solution of MFGs and have produced many excellent results. But most of them are directed against single-population. Specifically, the simultaneous pioneering works of MFGs theory are proposed by Lasry \& Lions [1]-[3] and Huang, Malhame \& Caines [4]-[6] independently. After that, some kinds of literature use rigorous analytical methods to study numerical algorithms in various situations, including continuous-state problems [35][47] and finite-state problems [48], [49]. In the absence of explicit solutions, there have been too many fast solvers for the solution of MFGs in two and three dimensions [50]-[55], but they all need grid-based spatial discretization. Grid-based numerical solutions will be cursed by dimensionality, namely, their computational complexity increases exponentially with the spatial dimension [32]. While the numerical solution method based on neural networks will be more suitable for the high-dimensional HJB-FPK coupled equations [33]. In terms of the numerical methods for solving high-dimensional MFGs, [31] perfectly avoids spatial grids of high-dimensional MFGs based on the machine learning framework, but their work is limited to the deterministic setting $(\sigma=0)$. Furthermore, [56] is the first document to solve the high-dimensional MFGs under a stochastic setting $(\sigma>0)$, which utilizes the natural connection between MFGs and generative adversarial neural networks (GANs) [57]. Based on the original technical difficulties (large-scale, high-dimensional), the multi-population model increases the complexity of sequential games, including heterogeneous group communication and two-level game reasoning. This makes the numerical framework for solving single-population MFGs no longer applicable. To sum up, for meeting the high requirements of more complex actual scene modeling, it is an urgent need to break through the numerical solution technology of multi-population high-dimensional stochastic MFGs.

Multi-population mean-field games, as an important subclass of MFGs, naturally arise in most practical applications [34]. Since the original contribution of [5], many researcher$\mathrm{s}$ have studied it by analytical methods, but the technical gap in the calculation of equilibrium solution is particularly prominent. [58] and [59] dealt with ergodic MFGs of multipopulation under different boundary conditions, including the existence and uniqueness results of systems. [19] and [21] 
consider mean field control problems with several populations, and the authors introduce these models for crowd motion (with local and non-local interactions respectively). They have studied optimality conditions and provided numerical results. [60] studies the issue of urban settlements and residential choice by using the MFG model to describe the interactions between two populations and provides some numerical simulations. [34] and [63] systematically study the problems of meanfield games and mean-field type control problems with multipopulation by analyzing the interaction of two populations. It can be seen that the above authors have studied the theory and application of multi-population MFGs, but the equilibrium solution parts generally adopt analytical methods, and their numerical examples are limited to low dimensions (ordinarily one-dimensional or two-dimensional). The numerical solution method of multi-population high-dimensional MFGs is still relatively blank.

Inspired by the above-mentioned cutting-edge works, in this paper, we are committed to solving the difficult problem of numerical solution for multi-population high-dimensional MFGs with stochasticity, which needs to take into account factors of game complexity, dimensionality disaster, and disturbances simultaneously.

\section{Main Contribution}

We present CA-Net, a coupled alternating neural network approach for tractably solving multi-population highdimensional MFGs in the stochastic case $(\sigma>0)$. To this end, we have made the following contributions.

1) We provide a general framework for modeling largescale heterogeneous multi-agent game systems, give a unified expression of multi-population MFGs under a two-layer game structure, and ensure the existence of equilibrium of such systems.

2) We generalize the potential variational primal-dual structure that MFGs exhibit and phrase the multi-population MFG problem as a convex-concave saddle-point problem. In this way, we circumvent spatial grids or uniformly sampling in high dimensions (the curse of dimensionality) and provide the cornerstone for the construction of the following solving network.

3) Based on the saddle-point problem, we parameterize the value functions and the density functions of multiple populations by two sets of neural networks, respectively. Then we design a generative adversarial network (GAN), a nonlinear coupled alternating neural network composed of multiple generators and multiple discriminators, that is, the solving network, referred to as CA-Net.

4) We simulate a classic task of heterogeneous MAS with numerical examples, verify and analyze the feasibility and effectiveness of multi-population high-dimensional stochastic MFG model and CA-Net algorithm. And the comparison with the baseline methods shows the advanced nature of our

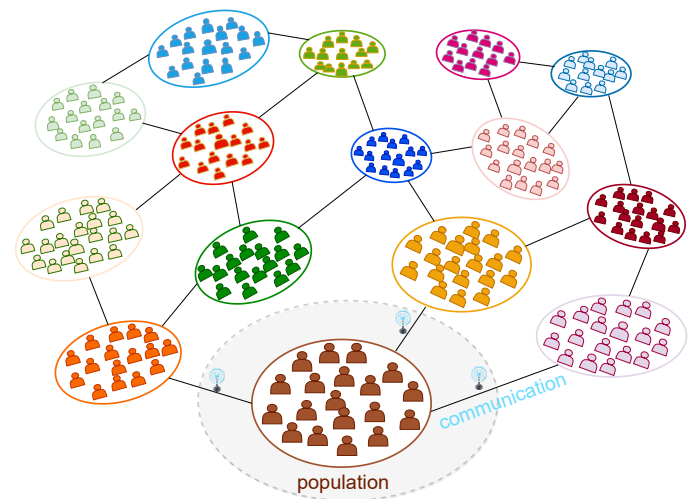

Fig. 1: Illustration of a multi-population model with local interactions. Large-scale agents are divided into different populations (indicated by different colors) according to different characteristics to participate in the game. The communication between populations follows the communication topology diagram (population is the vertex, and the communication is the black solid line). A representative continuum population (bottom shaded part) with small rational agents communicates with three neighboring populations to play a non-cooperative differential game on a time horizon.

method.

To the best of our knowledge, CA-Net is the first model that solves multi-population high-dimensional MFGs with stochasticity $(\sigma>0)$.

\section{System Model ANd Problem Formulation}

\section{A. Control Formulation of Multi-Population Model}

We consider a multi-agent system (MAS) with $N$ agents indexed by $i \in I=\{1, \ldots, N\}$, who take part in the game are not all homogeneous, and can be subdivided in $P$ agent populations indexed by $p(i) \in Q=\{1, \ldots, P\}$, as illustrated in Fig. 1. And each continuum population $p$ with small rational agents plays a non-cooperative differential game on a time horizon $[0, T]$. To model the set of $P$ distinct and interconnected populations, we assume a graph $G=(Q, E)$ is given, where $Q=\{1, \ldots, P\}$ is the set of vertices, one per each agent population, and $E \subseteq Q \times Q$ is the set of all weighted edges. More specifically, $\arg (p, q)$ belongs to $E$ if the corresponding weight $v_{p, q}>0$. A positive scalar weight $v_{p, q}$ means the relative influence that population $p$ assigns to population $q$. For example, $v_{p, q}$ could be calculated as the number of agents of population $q$ over the total number of agents of all the neighbor populations. Although most results are easily extended to more general graphs, possibly directed, time-varying, for the sake of simplicity, we henceforward assume that $G=(Q, E)$ is a connected undirected graph. We denote the set of neighbors of $p$ by $V(p)=\{q \in Q \mid(p, q) \in$ $E\}$, and the cardinality of the set $V(p)$ by $|V(p)|$.

$$
J_{p}\left(u^{p}, \rho^{p}, \rho^{-p}\right)=\mathbb{E}\left[\int_{0}^{T} L_{p}\left(x^{p}(t), u^{p}(t)\right)+F_{p}\left(x^{p}(t), \rho^{p}\left(x^{p}(t), t\right), \rho^{-p}\left(x^{-p}(t), t\right)\right) \mathrm{d} t+G_{p}\left(x^{p}(T), \rho^{p}\left(x^{p}(T), T\right), \rho^{-p}\left(x^{-p}(T), T\right)\right)\right]
$$


In the same agent population, we argue that the agents share a common characteristic (e.g. type, function, angle range, or upper bound of velocity). Each agent $i \in I$ belongs to a population $p(i) \in Q$ and is characterized by a state $x^{p}(t) \in \mathbb{R}^{n}$ at time $t \in[0, T]$. More precisely, we consider that the state of the generic player $i$ of population $p$ is a stochastic process $\left\{x_{p \in Q}^{p}\right\}$ in $\mathbb{R}^{P n}$ with the following forward McKean-Vlasov dynamics

$$
\mathrm{d} x^{p}=h^{p}\left(x^{p}, \rho^{p}, \rho^{-p}, u^{p}\right) \mathrm{d} t+\sigma^{p} \mathrm{~d} w^{p}
$$

where $u^{p}:[0, T] \rightarrow \mathcal{U}_{p} \subseteq \mathbb{R}^{m}$ is the control input (strategy), $\rho^{p}: \mathbb{R}^{n} \times[0, T] \rightarrow \mathcal{P}_{2}\left(\mathbb{R}^{n}\right)$ is the probability density function, $\rho^{-p}=\left(\rho^{q}\right)_{q \in V(p)}: \mathbb{R}^{n} \times[0, T] \rightarrow \mathcal{P}_{2}\left(\mathbb{R}^{n}\right)^{|V(p)|}$ describes the distribution vector of neighbor populations, $h^{p}$ : $\mathbb{R}^{n} \times \mathcal{P}_{2}\left(\mathbb{R}^{n}\right)^{|V(p)|+1} \times \mathcal{U}_{p} \rightarrow \mathbb{R}^{n}$ is the nonlinear evolution function, $\sigma^{p} \in \mathbb{R}^{n \times m}$ is a fixed coefficient matrix of population dependent volatility term, $w^{p}$ denotes an $m$-dimensional Wiener process (standard Brownian motion) comes from the environment, where each component $w_{k}^{p}$ is independent of $w_{l}^{p}$ for all $k \neq l, p=1, \ldots, P$.

Then, for the generic player $i$ of population $p, p=1, \ldots, P$, we consider the following cost function (2), as shown at the bottom of the previous page.

In Eq. (2), $L_{p}: \mathbb{R}^{n} \times \mathcal{U}_{p} \rightarrow \mathbb{R}$ is a running cost incurred by an agent based solely on their actions, $F_{p}: \mathbb{R}^{n} \times$ $\mathcal{P}_{2}\left(\mathbb{R}^{n}\right)^{|V(p)|+1} \rightarrow \mathbb{R}$ is a running cost incurred by an agent based on their interaction with rest of the same population and the neighbor populations, and $G_{p}: \mathbb{R}^{n} \times \mathcal{P}_{2}\left(\mathbb{R}^{n}\right)^{|V(p)|+1} \rightarrow \mathbb{R}$ is a terminal cost incurred by an agent based on their final state and the final distribution of the whole related populations. The terms $F$ and $G$ are called mean field terms because they encode the interaction of a single agent with the rest of the populations.

\section{B. The Formulation of Multi-Population Mean-Field Game}

Mean-field games (MFGs) are a class of problems that encode large populations of interacting agents into systems of coupled partial differential equations. As an important subclass of MFGs, multi-population mean-field game not only has the advantages of MFG, e.g., it overcomes the difficulties of largescale and information sharing, but allows for the representation of some more realistic situations, such as [60]-[62].

For every population $p=1, \ldots, P$, each agent $i$ of population $p(i)$ forecasts a distribution of the population $\left\{\rho^{p}(\cdot, t)\right\}_{t=0}^{T}$, and aims at minimizing their cost which eventually reaches the Nash equilibrium where no agent can reduce individual cost by changing its control strategy unilaterally. That is, for every $x^{p} \in \mathbb{R}^{n}$,

$$
J_{p}\left(\hat{u}^{p}, \rho^{p}, \rho^{-p}\right) \leq J_{p}\left(u^{p}, \rho^{p}, \rho^{-p}\right), \quad \forall u^{p}:[0, T] \rightarrow \mathcal{U}_{p}
$$

where $\hat{u}^{p}$ is the equilibrium strategy of an agent at state $x^{p}$. Here, we assume that agents are small, and their unilateral actions do not alter the density $\rho^{p}$. Literature [34] and [63] of multi-population MFG provide sufficient and necessary conditions for the solution to the coupled system of forwardbackward stochastic partial differential equations (SPDEs), respectively.

Remark 1: Under appropriate assumptions, the solution to the MFG provides an approximate Nash equilibrium for the corresponding game with a large but finite number of agents [34].

From Eq. (3), we have that each agent $i$ of population $p(i)$ solves an optimal control problem that has a value function

$$
\Phi_{p}\left(x^{p}, t\right)=\inf _{u^{p}} J_{p}\left(u^{p}, \rho^{p}, \rho^{-p}\right) \text {, s.t. Eq. (1) }
$$

From the optimal control theory (for details, see reference [64], sections 2.2, 2.3, and 3.4.2 or reference [63], section 4.1), we have that $\Phi_{p}$ solves the HJB equation (5), as shown at the bottom of the page.

where the Hamiltonian, $H_{p}: \mathbb{R}^{n} \times \mathcal{P}_{2}\left(\mathbb{R}^{n}\right)^{|V(p)|+1} \times \mathbb{R}^{n} \rightarrow$ $\mathbb{R}$, is defined as

$$
H_{p}\left(x^{p}, \rho^{p}, \rho^{-p}, z^{p}\right)=\sup _{u^{p}}\left\{-L_{p}\left(x^{p}, u^{p}\right)-z^{p \top} h^{p}\left(x^{p}, \rho^{p}, \rho^{-p}, u^{p}\right)\right\}
$$

Hence we have

$$
\begin{gathered}
z^{p}=\nabla_{x^{p}} \Phi_{p}\left(x^{p}, t\right) \\
h^{p}\left(x^{p}, \rho^{p}, \rho^{-p}, \hat{u}^{p}\right)=-\nabla_{z^{p}} H_{p}\left(x^{p}, \rho^{p}, \rho^{-p}, \nabla \Phi_{p}\left(x^{p}, t\right)\right)
\end{gathered}
$$

According to Ito's lemma (for details, see reference [64], sections 2.2 and 3.2.2), $\rho^{p}$ satisfies the forward equation of $x^{p}$, i.e. the FPK equation (9), as shown at the bottom of the page, where $\rho_{0}^{p} \in \mathcal{P}_{2}\left(\mathbb{R}^{n}\right)$ is the given population density at time $t=0$.

The above discussion provides an alternative to optimizing the strategy $u^{p}$ individually for each agent of the multipopulation model. One can obtain the optimal strategy for all agents of multi-population simultaneously by solving the coupled SPDEs system given by the HJB (5) and the FPK (9). Following this macroscopic approach, our first step is to accurately establish a multi-population agent model and reduce the immense computational challenges and communication energy consumption caused by heterogeneous multi-agent systems.

$$
\mathrm{HJB}: \quad \begin{aligned}
-\partial_{t} \Phi_{p}\left(x^{p}, t\right)-\frac{\sigma^{p 2}}{2} \Delta \Phi_{p}\left(x^{p}, t\right)+H_{p}\left(x^{p}, \rho^{p}, \rho^{-p}, \nabla \Phi_{p}\left(x^{p}, t\right)\right) & =F_{p}\left(x^{p}, \rho^{p}\left(x^{p}, t\right), \rho^{-p}\left(x^{-p}, t\right)\right) \\
\Phi_{p}\left(x^{p}, T\right) & =G_{p}\left(x^{p}, \rho^{p}\left(x^{p}, T\right), \rho^{-p}\left(x^{-p}, T\right)\right)
\end{aligned}
$$

FPK: $\quad \partial_{t} \rho^{p}\left(x^{p}, t\right)-\frac{\sigma^{p 2}}{2} \Delta \rho^{p}\left(x^{p}, t\right)-\operatorname{div}\left(\rho^{p}\left(x^{p}, t\right) \nabla_{z^{p}} H_{p}\left(x^{p}, \rho^{p}, \rho^{-p}, \nabla \Phi_{p}\left(x^{p}, t\right)\right)\right)=0$

$$
\rho^{p}\left(x^{p}, 0\right)=\rho_{0}^{p}\left(x^{p}\right)
$$


Remark 2: Instead of including all agents' states in the value function [65]-[67], the MFGs' deterministic flows of probability density describe the population's current information. Utilizing the density distribution can benefit from two aspects: 1) the dimension of the density distribution is independent of the number of agents so the dimension disaster is overcome theoretically and 2) the density distribution can be obtained by locally solving the FPK equation so the information sharing between all agents is not necessary.

Remark 3: From an engineering angle, the proposed multipopulation MFG model can provide an accurate and feasible scheme for the large-scale heterogeneous MAS. Because it breaks the dimensionality curse of the system theoretically and ensures the Nash equilibrium solution under the limit conditions.

To compute the optimal control policy of the large-scale heterogeneous MAS, the mean-field equations (5) and (9) need to be solved in real-time. However, the HJB and FPK are two high-dimensional nonlinear SPDEs that are also solved in different directions. Therefore, the analytical solution is nearly impossible to obtain. In this article, a novel CA-Net algorithm induced from the idea of the generative adversarial network (GAN) is developed to find the solution of the coupled HJBFPK equations in high dimensions.

\section{GAN-BASED APPROACH FOR HIGH-DIMENSIONAL SPDES OF MULTI-POPULATION PROBlEM}

We phrase the multi-population MFG problem as a saddlepoint problem and parameterize the value functions and the density functions of multi-population, then use a coupled alternating neural network (CA-Net) to find the equilibrium solution.

\section{A. Variational Primal-Dual Formulation of Multi-Population Mean-Field Game}

According to the natural connection between MFGs and GANs, we deduce the multi-population MFG formulation to a primal-dual convex-concave one, then can obtain a tailored coupled alternating network to find the equilibrium solution.

An multi-population MFG system consists of the two equations (5) and (9) is called potential, if there exist functionals $\mathcal{F}_{p}, \mathcal{G}_{p}: \mathcal{P}_{2}\left(\mathbb{R}^{n}\right) \rightarrow \mathbb{R}, p=1, \ldots, P$ such that

$$
\delta_{\rho^{p}} \mathcal{F}_{p}=F_{p}\left(x^{p}, \rho^{p}, \rho^{-p}\right) \quad \text { and } \quad \delta_{\rho^{p}} \mathcal{G}_{p}=G_{p}\left(x^{p}, \rho^{p}, \rho^{-p}\right)
$$

where

$$
\begin{aligned}
\int_{\mathbb{R}^{n}} F_{p}\left(x^{p}, \rho^{p}\right) \mu\left(x^{p}\right) \mathrm{d} x^{p} & =\lim _{h \rightarrow 0} \frac{\mathcal{F}_{p}\left(\rho^{p}+h \mu\right)-\mathcal{F}_{p}\left(\rho^{p}\right)}{h}, \forall \mu \\
\int_{\mathbb{R}^{n}} G_{p}\left(x^{p}, \rho^{p}\right) \mu\left(x^{p}\right) \mathrm{d} x^{p} & =\lim _{h \rightarrow 0} \frac{\mathcal{G}_{p}\left(\rho^{p}+h \mu\right)-\mathcal{G}_{p}\left(\rho^{p}\right)}{h}, \forall \mu
\end{aligned}
$$

That is, there exist functionals $\mathcal{F}, \mathcal{G}$ such that their variational derivatives with respect to $\rho$ are the interaction and terminal costs $F$ and $G$ from (5) and (9). A key feature of potential MFG is that it can be equivalently expressed as a convexconcave saddle point optimization problem. So the multipopulation MFG problem in (5) and (9) can be reformulate as a variational problem [3], [51]. For the generic player $i$ of population $p$, we get:

$$
\begin{aligned}
& \inf _{\rho^{p}, u^{p}} \int_{0}^{T} \int_{\mathbb{R}^{n}} \rho^{p}\left(x^{p}, t\right) L_{p}\left(x^{p}(t), u^{p}(t)\right) \mathrm{d} x^{p} \mathrm{~d} t \\
& \quad+\int_{0}^{T} \mathcal{F}_{p}\left(x^{p}(t), \rho^{p}\left(x^{p}(t), t\right), \rho^{-p}\left(x^{-p}(t), t\right)\right) \mathrm{d} t \\
& \quad+\mathcal{G}_{p}\left(x^{p}(T), \rho^{p}\left(x^{p}(T), T\right), \rho^{-p}\left(x^{-p}(T), T\right)\right) \\
& \text { s.t. } \partial_{t} \rho^{p}-\frac{\sigma^{p 2}}{2} \Delta \rho^{p}+\nabla \cdot\left(\rho^{p} h^{p}\right)=0, \quad \rho^{p}\left(x^{p}, 0\right)=\rho_{0}^{p}\left(x^{p}\right) \\
& \quad \partial_{t} \rho^{q}-\frac{\sigma^{q 2}}{2} \Delta \rho^{q}+\nabla \cdot\left(\rho^{q} h^{q}\right)=0, \quad \rho^{q}\left(x^{q}, 0\right)=\rho_{0}^{q}\left(x^{q}\right) \\
& \quad \rho^{-p}=\left(\rho^{q}\right)_{q \in V(p)}
\end{aligned}
$$

Now, the running cost $L_{p}$ could be regarded as the Lagrangian function corresponding to the Legendre transform of the Hamiltonian $H_{p}(6)$, and $\mathcal{F}_{p}, \mathcal{G}_{p}$ are mean field interaction terms corresponding to $F_{p}, G_{p}$ in (2).

In order to obtain the Lagrangian dual problem of (10) [62], [68], we start with introducing the value function $\Phi_{p}\left(x^{p}, t\right)$ as a Lagrange multiplier, then insert the constraint (FPK equations) into the objective (cost function) to get the following extended cost function:

$$
\begin{aligned}
\sup _{\Phi_{p}} \inf _{\rho^{p}, u^{p}} & \int_{0}^{T} \int_{\mathbb{R}^{n}} \rho^{p}\left(x^{p}, t\right) L_{p}\left(x^{p}(t), u^{p}(t)\right) \mathrm{d} x^{p} \mathrm{~d} t \\
& +\int_{0}^{T} \mathcal{F}_{p}\left(x^{p}(t), \rho^{p}\left(x^{p}(t), t\right), \rho^{-p}\left(x^{-p}(t), t\right)\right) \mathrm{d} t \\
& +\mathcal{G}_{p}\left(x^{p}(T), \rho^{p}\left(x^{p}(T), T\right), \rho^{-p}\left(x^{-p}(T), T\right)\right) \\
& -\int_{0}^{T} \int_{\Omega} \Phi_{p}\left(x^{p}, t\right)\left(\partial_{t} \rho^{p}-\frac{\sigma^{p 2}}{2} \Delta \rho^{p}\right. \\
& \left.+\nabla \cdot\left(\rho^{p}\left(x^{p}, t\right) h^{p}\left(x^{p}, \rho^{p}, \rho^{-p}, u^{p}\right)\right)\right) \mathrm{d} x^{p} \mathrm{~d} t \\
& -\sum_{q \in V(p)} \int_{0}^{T} \int_{\Omega} \Phi_{q}\left(x^{q}, t\right)\left(\partial_{t} \rho^{q}-\frac{\sigma^{q 2}}{2} \Delta \rho^{q}\right. \\
& \left.+\nabla \cdot\left(\rho^{q}\left(x^{q}, t\right) h^{q}\left(x^{q}, \rho^{q}, \rho^{-q}, u^{q}\right)\right)\right) \mathrm{d} x^{q} \mathrm{~d} t
\end{aligned}
$$

Following the approach of [51], [69], [70], we write the constraint $\partial_{t} \rho^{p}-\frac{\sigma^{p 2}}{2} \Delta \rho^{p}+\nabla \cdot\left(\rho^{p} h^{p}\right)=0, p=1, \ldots, P$ in weak form, i.e.,

$$
\begin{aligned}
& \int_{0}^{T} \int_{\mathbb{R}^{n}} \rho^{p} \partial_{t} \Phi_{p}+\frac{\sigma^{p 2}}{2} \Delta \Phi_{p} \rho^{p}+\nabla \Phi_{p} \cdot\left(\rho^{p} h^{p}\right) \mathrm{d} x^{p} \mathrm{~d} t \\
& +\int_{\mathbb{R}^{n}} \Phi_{p}\left(x^{p}, 0\right) \rho_{0}^{p}\left(x^{p}\right) \mathrm{d} x^{p}-\int_{\mathbb{R}^{n}} \Phi_{p}\left(x^{p}, T\right) \rho^{p}\left(x^{p}, T\right) \mathrm{d} x^{p}=0
\end{aligned}
$$

Finally, utilizing (12) and integrating by parts, we can rewrite our problem as

$$
\begin{aligned}
\underset{\rho^{p}}{\sin _{\Phi_{p}}} \sup _{0} \int_{\mathbb{R}^{n}}^{T}\left(\partial_{t} \Phi_{p}+\frac{\sigma^{p 2}}{2} \Delta \Phi_{p}-H_{p}\left(x^{p}, \nabla \Phi_{p}\right)\right) \rho^{p}\left(x^{p}, t\right) \mathrm{d} x^{p} \mathrm{~d} t \\
+\int_{0}^{T} \mathcal{F}_{p}\left(x^{p}(t), \rho^{p}\left(x^{p}(t), t\right), \rho^{-p}\left(x^{-p}(t), t\right)\right) \mathrm{d} t \\
+ \\
+\mathcal{G}_{p}\left(x^{p}(T), \rho^{p}\left(x^{p}(T), T\right), \rho^{-p}\left(x^{-p}(T), T\right)\right) \\
+\int_{\mathbb{R}^{n}} \Phi_{p}\left(x^{p}, 0\right) \rho_{0}^{p}\left(x^{p}\right) \mathrm{d} x^{p}-\int_{\mathbb{R}^{n}} \Phi_{p}\left(x^{p}, T\right) \rho^{p}\left(x^{p}, T\right) \mathrm{d} x^{p} \\
+\sum_{q \in V(p)}\left\{\int_{0}^{T} \int_{\mathbb{R}^{n}}\left(\partial_{t} \Phi_{q}+\frac{\sigma^{q}}{2} \Delta \Phi_{q}+\nabla \Phi_{q} \cdot h^{q}\right)\right. \\
\quad \times \rho^{q}\left(x^{q}, t\right) \mathrm{d} x^{q} \mathrm{~d} t+\int_{\mathbb{R}^{n}} \Phi_{q}\left(x^{q}, 0\right) \rho_{0}^{q}\left(x^{q}\right) \mathrm{d} x^{q} \\
\\
\left.-\int_{\mathbb{R}^{n}} \Phi_{q}\left(x^{q}, T\right) \rho^{q}\left(x^{q}, T\right) \mathrm{d} x^{q}\right\}
\end{aligned}
$$

This formula can also be deduced in the context of HJB equations in probability measure spaces [52], or by integrating the HJB (5) and the FPK (9) equations for $\rho^{p}$ and $\Phi_{p}$, respectively [71]. 
In [71], it has been shown that all MFG systems coincide with an infinite-dimensional two-player general-sum game, and the potential MFGs are the ones that correspond to zerosum games. In its elaboration, player 1 represents the whole population and their strategy is the population's probability distribution. While player 2 represents the generic agent and their strategy is the value function. The goal of player 2 is to provide a strategy that yields the best response of a generic agent against the population. This elaboration is consisten$\mathrm{t}$ with the intuition behind generative adversarial networks (GANs). By drawing from a natural connection between MFGs and GANs, [56] presents APAC-Net, an alternating population and agent control neural network, which is the first model for solving high-dimensional MFGs of a single population in the stochastic case. We extended this methodology to highdimensional MFGs of multiple characteristic populations in the stochastic setting $\left(\sigma^{p}>0\right)$ and solve the HJB-FPK coupled system (5)-(9) for a certain population $p$.

Remark 4: Under mild assumptions on $\mathcal{F}_{p}$ and $\mathcal{G}_{p}$, each spatial integral is really an expectation from $\rho^{p}, p=1, \ldots, P$. The formulation (13) is the cornerstone of our approach.

\section{B. $C A-N e t$}

Recall that from Riemann integral to Lebesgue integral, the former focuses on grid-points of the definition domain, while the latter focuses on values of the function. The proposed CA-Net does not discretize the domain and solve for the function values on grid-points, but avoids these problems by parameterizing the function and solving for the function itself. This is also the fundamental reason why CA-Net can overcome the dimensional disaster of MFG equations. Another important point is that although the idea is inspired by GANs, and the training process of single-population MFG solving networks is analog to that of GANs (a single generator and discriminator). For multi-population MFG solving network, it is a nonlinear coupled alternating neural network formed by multiple generators and multiple discriminators. We show the structure and training process of our CA-Net in the diagram Fig. 2.

We initialize $P$ pairs of neural networks $N_{\omega}^{p}\left(x^{p}, t\right)$ and $N_{\theta}^{p}\left(y^{p}, t\right), p=1, \ldots, P$. We then let

$$
\begin{aligned}
& \Phi_{\omega}^{p}\left(x^{p}, t\right)=(1-t) N_{\omega}^{p}\left(x^{p}, t\right)+t G_{p}\left(x^{p}\right) \\
& \mathrm{G}_{\theta}^{p}\left(y^{p}, t\right)=(1-t) y^{p}+t N_{\theta}^{p}\left(y^{p}, t\right)
\end{aligned}
$$

where $y^{p} \sim \rho_{0}^{p}$ are samples drawn from the initial distribution, and $\mathrm{G}_{\theta}^{p}$ produces samples from $\rho_{0}^{p}$ at $t=0$. While, we set $\rho^{p}(\cdot, t)=\mathrm{G}_{\theta}^{p}(\cdot, t) \# \rho_{0}^{p}$, it is the push-forward of $\rho_{0}^{p}$. In this setting, we train $\mathrm{G}_{\theta}^{p}(\cdot, t)$ to produce samples from $\rho^{p}(\cdot, t)$. We note that $\Phi_{\omega}^{p}$ and $\mathrm{G}_{\theta}^{p}$ automatically satisfy the terminal and initial condition, respectively.

Our approach for training neural networks includes parallel alternately training $P$ pairs of $\mathrm{G}_{\theta}^{p}$ (the density distribution of population $p$ ) and $\Phi_{\omega}^{p}$ (the value function for the generic agent of population $p$ ). Intuitively, in order to obtain the equilibrium of multi-population MFG, we are training a coupled alternating neural networks (CA-Net) about multi-group distributions and agent controls. Specifically, we train $\Phi_{\omega}^{p}$ by

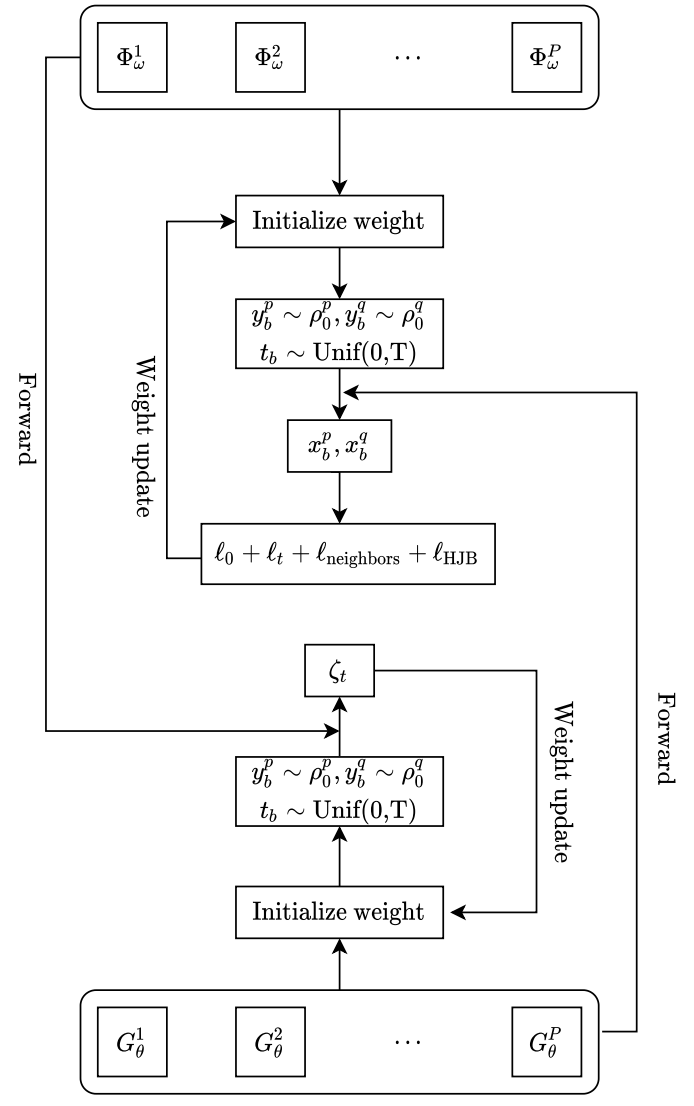

Fig. 2: Visualization of the structure and training process of CA-Net. CA-Net is composed of multiple generators (Bottom) and multiple discriminators (Top). Its training process is divided into two coupled alternating training parts. Initialize the weights of $\Phi_{\omega}^{p}$ and $\mathrm{G}_{\theta}^{p}, p=1, \ldots, P$ randomly, the first step is to train the value function network $\Phi_{\omega}^{p}$, batch a random sample points $y_{b}^{p}, y_{b}^{q}, t_{b}, b=1, \ldots, B$, get $x_{b}^{p}, x_{b}^{q}$ forward through the density function network $\mathrm{G}_{\theta}^{p}$. The weight $\omega$ of $\Phi_{\omega}^{p}$ is updated by the gradient descent algorithm to minimize the loss function $\ell_{0}+\ell_{t}+\ell_{\text {neighbors }}+\ell_{\text {HJB }}$. The second step is to train the density function network $\mathrm{G}_{\theta}^{p}$, batch a random sample points $y_{b}^{p}, y_{b}^{q}, t_{b}, b=1, \ldots, B$, update the weight $\theta$ of $\mathrm{G}_{\theta}^{p}$ through the value function network $\Phi_{\omega}^{p}$ and the gradient descent algorithm to minimize the loss function $\zeta_{t}$. Then loop the two parts to achieve the optimal result.

first sampling a batch $\left\{y_{b}^{p}\right\}_{b=1}^{B}$ from the given initial density $\rho_{0}^{p}$, and $\left\{t_{b}\right\}_{b=1}^{B}$ uniformly from $[0,1]$, i.e. we are sampling from $\rho_{0}^{p} \times \operatorname{Unif}([0,1])$. Next, we compute the push-forward states $x_{b}^{p}=\mathrm{G}_{\theta}^{p}\left(y_{b}^{p}, t_{b}\right)$ for $b=1, \ldots, B$. It should be emphasized that we construct the loss functions of CA-Net according to the variational primal-dual formulation (13) of multi-population MFG. So, here is the main loss item for training the discriminator,

$$
\begin{aligned}
\operatorname{loss}_{\Phi^{p}} & =\frac{1}{B} \sum_{b=1}^{B} \Phi_{\omega}^{p}\left(x_{b}^{p}, 0\right)+\frac{1}{B} \sum_{b=1}^{B}\left\{\partial_{t} \Phi_{\omega}^{p}\left(x_{b}^{p}, t_{b}\right)\right. \\
& \left.+\frac{\sigma^{p}}{2} \Delta \Phi_{\omega}^{p}\left(x_{b}^{p}, t_{b}\right)-H_{p}\left(\nabla_{x^{p}} \Phi_{\omega}^{p}\left(x_{b}^{p}, t_{b}\right)\right)\right\}
\end{aligned}
$$


Of course, we need to consider adding a dual penalty term from neighbors.

$$
\begin{aligned}
\text { penalty }_{\text {neighbors }} & =\eta \sum_{q \in V(p)}\left\{\frac { 1 } { B } \sum _ { b = 1 } ^ { B } \left[\partial_{t} \Phi_{\omega}^{q}\left(x_{b}^{q}, t_{b}\right)+\frac{\sigma^{q 2}}{2} \Delta \Phi_{\omega}^{q}\left(x_{b}^{q}, t_{b}\right)\right.\right. \\
& \left.\left.+\nabla_{x^{q}} \Phi_{\omega}^{q}\left(x_{b}^{q}, t_{b}\right) \cdot h^{q}\left(x_{b}^{q}, t_{b}\right)\right]\right\}
\end{aligned}
$$

And we can optionally add a regularization term to penalize deviations from the HJB equations [31], [72].

$$
\begin{aligned}
\text { penalty }_{\mathrm{HJB}} & =\lambda \frac{1}{B} \sum_{b=1}^{B} \| \partial_{t} \Phi_{\omega}^{p}\left(x_{b}^{p}, t_{b}\right)+\frac{\sigma^{p 2}}{2} \Delta \Phi_{\omega}^{p}\left(x_{b}^{p}, t_{b}\right) \\
& -H_{p}\left(\nabla_{x^{p}} \Phi_{\omega}^{p}\left(x_{b}^{p}, t_{b}\right)\right)+F_{p}\left(x_{b}^{p}, t_{b}\right) \|
\end{aligned}
$$

This extra regularization term has also been found effective in, e.g., Wasserstein GANs [73]. Finally, we backpropagate the loss to the weights of $\Phi_{\omega}^{p}$. To train the generator, we again sample $\left\{y_{b}^{p}\right\}_{b=1}^{B}$ and $\left\{t_{b}\right\}_{b=1}^{B}$ as before, and compute

$$
\begin{aligned}
\operatorname{loss}_{\mathrm{G}^{p}} & =\frac{1}{B} \sum_{b=1}^{B}\left\{\partial_{t} \Phi_{\omega}^{p}\left(\mathrm{G}_{\theta}^{p}\left(y_{b}^{p}\right), t_{b}\right)+\frac{\sigma^{p 2}}{2} \Delta \Phi_{\omega}^{p}\left(\mathrm{G}_{\theta}^{p}\left(y_{b}^{p}\right), t_{b}\right)\right. \\
& \left.-H_{p}\left(\nabla_{x} p \Phi_{\omega}^{p}\left(\mathrm{G}_{\theta}^{p}\left(y_{b}^{p}\right), t_{b}\right)\right)+F_{p}\left(\mathrm{G}_{\theta}^{p}\left(y_{b}^{p}\right), t_{b}\right)\right\}
\end{aligned}
$$

Finally, we backpropagate this loss to the weights of $\mathrm{G}_{\theta}^{p}$. Please refer to Algorithm 1 for detailed operation flow.

\section{NUMERICAL EXPERIMENTS}

In this section, We demonstrate the potential of the proposed CA-Net on large-scale heterogeneous MAS control problems. First, we show the feasibility of using multi-population MFG to model large-scale heterogeneous MAS and the convergence of using CA-Net to solve the corresponding problems in highdimensional space in Sec. IV-A. Second, we also illustrate the behaviors of the multi-population MFG solutions for different values of stochastic characteristic $\sigma$ in Sec. IV-B. Third, we further reveal the relationship between algorithm parameters and costs in Sec. IV-C. Last, to demonstrate the competitiveness of our approach, we compare the CA-Net algorithm's performance against baseline methods in Sec. IVD.

\section{A. Multi-Group Quadcopters Trajectory Planning Example}

This experiment is inspired by [56], [74], [75], we examine a realistic scenario where the dynamics are that of a quadcopter (a.k.a. quadrotor craft), which is an aerial vehicle with four rotary wings (similar to many of the consumer drones seen today), and utilizes its four rotors to propel itself across space from an initial state to target state. The designed test environment is a 3-D real flight space and has a total of a thousand drones, which are divided into five populations with different velocity ranges to perform tasks-flying from their initial distribution to the common terminal distribution. Here, graph $G=(Q, E)$ is considered as a global communication between groups. The dynamics of the generic quadrotor craft $i$ of population $p, p=1, \ldots, P$, are given as,

$$
\left\{\begin{array}{l}
\ddot{x}_{p}=\frac{u_{p}}{m_{p}}\left(\sin \left(\phi_{p}\right) \sin \left(\psi_{p}\right)+\cos \left(\phi_{p}\right) \cos \left(\psi_{p}\right) \sin \left(\theta_{p}\right)\right) \\
\ddot{y}_{p}=\frac{u_{p}}{m_{p}}\left(-\cos \left(\psi_{p}\right) \sin \left(\phi_{p}\right)+\cos \left(\phi_{p}\right) \sin \left(\theta_{p}\right) \sin \left(\psi_{p}\right)\right) \\
\ddot{z}_{p}=\frac{u_{p}}{m_{p}} \cos \left(\theta_{p}\right) \cos \left(\phi_{p}\right)-g \\
\ddot{\psi}_{p}=\tilde{\tau}_{\psi_{p}} \\
\ddot{\theta}_{p}=\tilde{\tau}_{\theta_{p}} \\
\ddot{\phi}_{p}=\tilde{\tau}_{\phi_{p}}
\end{array}\right.
$$

where $x_{p}, y_{p}$, and $z_{p}$ are the usual Euclidean spatial coordinates of the quadcopter, and $\phi_{p}, \theta_{p}$ and $\psi_{p}$ are the angular coordinates of roll, pitch, and yaw, respectively. The constant $m_{p}$ is the quadcopter's mass, and $g$ is the gravitation acceleration constant on Earth. The variables $u_{p}, \tilde{\tau}_{\psi_{p}}, \tilde{\tau}_{\theta_{p}}, \tilde{\tau}_{\phi_{p}}$ are the controls representing thrust, and angular acceleration, respectively [76]. In order to fit a control framework, the above second-order system is turned into a first-order system:

$$
\left\{\begin{array}{l}
\dot{x}_{p}=v_{x_{p}} \\
\dot{y}_{p}=v_{y_{p}} \\
\dot{z}_{p}=v_{z_{p}} \\
\dot{\psi}_{p}=v_{\psi_{p}} \\
\dot{\theta}_{p}=v_{\theta_{p}} \\
\dot{\phi}_{p}=v_{\phi_{p}} \\
\dot{v}_{x_{p}}=\frac{u_{p}}{m_{p}} h_{7}\left(\psi_{p}, \theta_{p}, \phi_{p}\right) \\
\dot{v}_{y_{p}}=\frac{u_{p}}{m_{p}} h_{8}\left(\psi_{p}, \theta_{p}, \phi_{p}\right) \\
\dot{v}_{z_{p}}=\frac{u_{p}}{m_{p}} h_{9}\left(\theta_{p}, \phi_{p}\right)-g \\
\dot{v}_{\psi_{p}}=\tilde{\tau}_{\psi_{p}} \\
\dot{v}_{\theta_{p}}=\tilde{\tau}_{\theta_{p}} \\
\dot{v}_{\phi_{p}}=\tilde{\tau}_{\phi_{p}}
\end{array}\right.
$$

where

$$
\left\{\begin{array}{l}
h_{7}\left(\psi_{p}, \theta_{p}, \phi_{p}\right)=\sin \left(\phi_{p}\right) \sin \left(\psi_{p}\right)+\cos \left(\phi_{p}\right) \cos \left(\psi_{p}\right) \sin \left(\theta_{p}\right) \\
h_{8}\left(\psi_{p}, \theta_{p}, \phi_{p}\right)=-\cos \left(\psi_{p}\right) \sin \left(\phi_{p}\right)+\cos \left(\phi_{p}\right) \sin \left(\theta_{p}\right) \sin \left(\psi_{p}\right) \\
h_{9}\left(\theta_{p}, \phi_{p}\right)=\cos \left(\theta_{p}\right) \cos \left(\phi_{p}\right)
\end{array}\right.
$$

which we will compactly denote as $\dot{\mathbf{x}}^{p}=\mathbf{h}^{p}\left(\mathbf{x}^{p}, \mathbf{u}^{p}\right)$, where $\mathbf{h}^{p}$ is a 12-dimensional vector function in the right-hand side of (20), $\mathbf{x}^{p}=\left[x_{p}, y_{p}, z_{p}, \psi_{p}, \theta_{p}, \phi_{p}, v_{x_{p}}, v_{y_{p}}, v_{z_{p}}, v_{\psi_{p}}, v_{\theta_{p}}, v_{\phi_{p}}\right]^{\top}$ $\in \mathbb{R}^{12}$ is the state with velocities $\mathbf{v}^{p}=\left[v_{x_{p}}, v_{y_{p}}, v_{z_{p}}, v_{\psi_{p}}, v_{\theta_{p}}\right.$, $\left.v_{\phi_{p}}\right]^{\top} \in \mathbb{R}^{6}$, and $\mathbf{u}^{p}=\left[u_{p}, \tilde{\tau}_{\psi_{p}}, \tilde{\tau}_{\theta_{p}}, \tilde{\tau}_{\phi_{p}}\right]^{\top} \in \mathbb{R}^{4}$ is the control. As can be seen, (20) is a 12-dimensional system that is highly coupled, nonlinear and high-dimensional. In the stochastic case, we add a noise term to the dynamics: $\mathrm{d} \mathbf{x}^{p}=\mathbf{h}^{p}\left(\mathbf{x}^{p}, \mathbf{u}^{p}\right) \mathrm{d} t+\sigma^{p} \mathrm{~d} \mathbf{W}_{t}^{p}$, where $\mathbf{W}$ denotes a Wiener process (standard Brownian motion). The interpretation here is that we are modeling the situation when the quadcopter suffers from noisy measurements.

For the Lagrangian cost function, we consider

$$
\begin{aligned}
L_{p}\left(\mathbf{u}^{p}\right) & =\frac{1}{2}\left\|\mathbf{u}^{p}(t)\right\|_{2}^{2} \\
& =\frac{1}{2}\left(u_{p}^{2}(t)+\tilde{\tau}_{\psi_{p}}^{2}(t)+\tilde{\tau}_{\theta_{p}}^{2}(t)+\tilde{\tau}_{\phi_{p}}^{2}(t)\right)
\end{aligned}
$$

We consider the Hamiltonian in (6) where $\mathbf{z}^{\mathbf{p}}=\left[z_{1}^{p}, z_{2}^{p}\right.$, $\left.\ldots, z_{12}^{p}\right]^{\top} \in \mathbb{R}^{12}$. Noting the optimality conditions of $(6)$ for the quadcopter problem are obtained by

$$
\begin{array}{r}
-\nabla_{\mathbf{u}^{p}} L_{p}\left(\mathbf{u}^{p}\right)-\mathbf{z}^{\mathbf{p}} \nabla_{\mathbf{u}^{p} \mathbf{h}^{p}}=\mathbf{0} \\
\left.\Rightarrow-\left[\begin{array}{c}
u_{p} \\
\tilde{\tau}_{\psi_{p}} \\
\tilde{\tau}_{\theta_{p}} \\
\tilde{\tau}_{\phi_{p}}
\end{array}\right]-\left[\begin{array}{c}
z_{7}^{p} \\
z_{8}^{p} \\
z_{9}^{p} \\
z_{10}^{p} \\
z_{11}^{p} \\
z_{12}^{p}
\end{array}\right] \begin{array}{cccc}
h_{7} / m_{p} & 0 & 0 & 0 \\
h_{8} / m_{p} & 0 & 0 & 0 \\
h_{9} / m_{p} & 0 & 0 & 0 \\
0 & 1 & 0 & 0 \\
0 & 0 & 1 & 0 \\
0 & 0 & 0 & 1
\end{array}\right]=\mathbf{0} \\
\Rightarrow-\left[\begin{array}{c}
u_{p} \\
\tilde{\tau}_{\psi_{p}} \\
\tilde{\tau}_{\theta_{p}} \\
\tilde{\tau}_{\phi_{p}}
\end{array}\right]-\left[\begin{array}{c}
\frac{1}{m_{p}}\left(z_{7}^{p} h_{7}+z_{8}^{p} h_{8}+z_{9}^{p} h_{9}\right) \\
z_{10}^{p} \\
z_{11}^{p} \\
z_{12}^{p}
\end{array}\right]=\mathbf{0}
\end{array}
$$

we can derive an expression for the controls as

$$
\begin{gathered}
u_{p}=-\frac{1}{m_{p}}\left(z_{7}^{p} h_{7}+z_{8}^{p} h_{8}+z_{9}^{p} h_{9}\right), \\
\tilde{\tau}_{\psi_{p}}=-z_{10}^{p}, \tilde{\tau}_{\theta_{p}}=-z_{11}^{p}, \tilde{\tau}_{\phi_{p}}=-z_{12}^{p}
\end{gathered}
$$




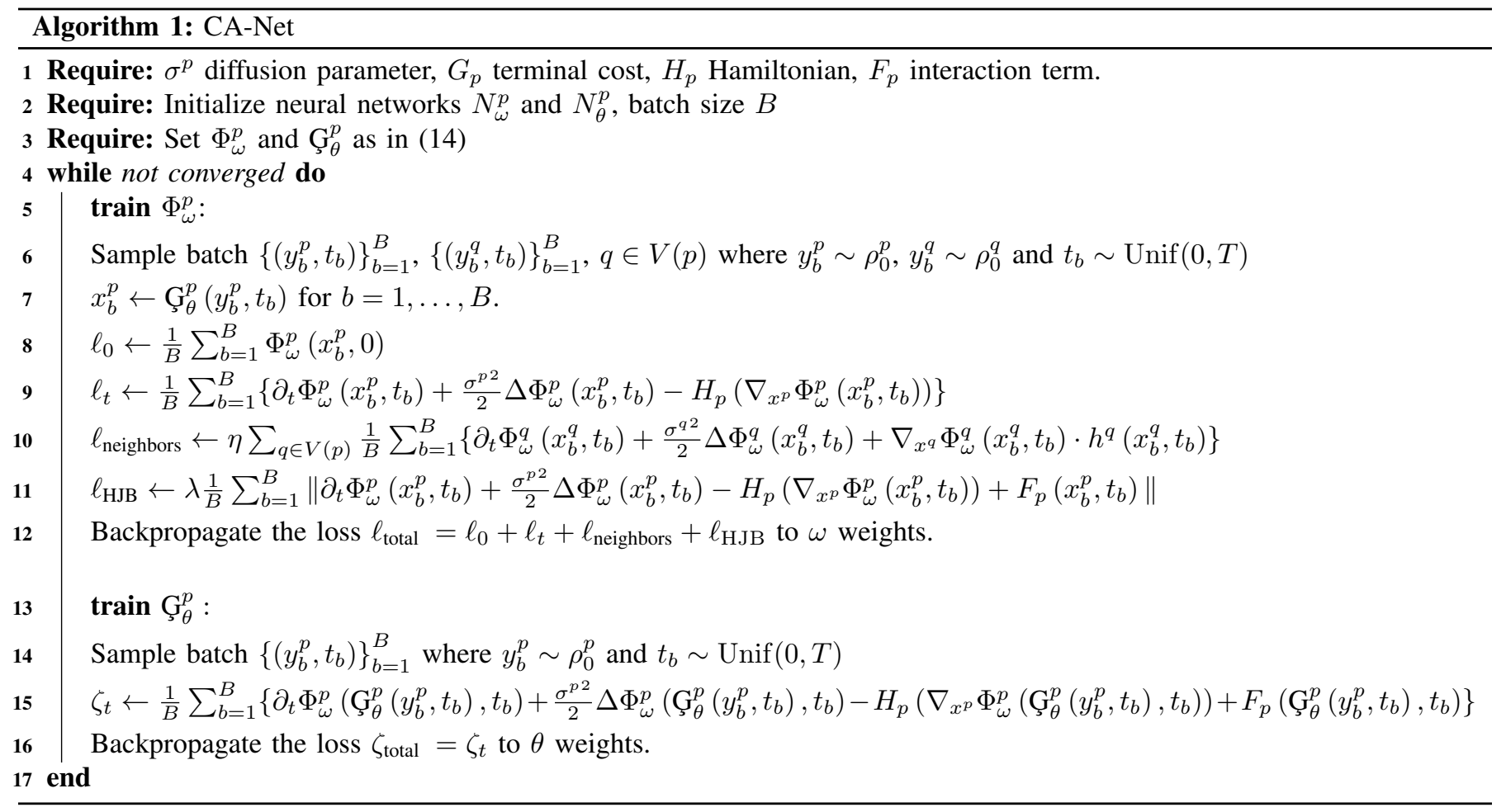

We therefore can compute the Hamiltonian

$$
\begin{aligned}
& H_{p}\left(t, \mathbf{x}^{p}, \mathbf{z}^{\mathbf{p}}\right)=-L_{p}\left(\mathbf{u}^{p}\right)-\left[v_{x_{p}} v_{y_{p}} v_{z_{p}}\right]\left[\begin{array}{c}
z_{1}^{p} \\
z_{2}^{p} \\
z_{3}^{p}
\end{array}\right] \\
& -\left[v_{\psi_{p}} v_{\theta_{p}} v_{\phi_{p}}\right]\left[\begin{array}{c}
z_{4}^{p} \\
z_{5}^{p} \\
z_{6}^{p}
\end{array}\right]+\frac{1}{m_{p}^{2}}\left(z_{7}^{p} h_{7}+z_{8}^{p} h_{8}+z_{9}^{p} h_{9}\right)^{2} \\
& +z_{9}^{p} g+\left(z_{10}^{p 2}+z_{11}^{p}{ }^{2}+z_{12}^{p}{ }^{2}\right)
\end{aligned}
$$

Finally, using (7) and (24), we compute the controls $\mathbf{u}^{p}$ using the CA-Net with

$$
\begin{gathered}
u_{p}=-\frac{1}{m_{p}}\left(h_{7} \frac{\partial \Phi_{p}}{\partial v_{x_{p}}}+h_{8} \frac{\partial \Phi_{p}}{\partial v_{y_{p}}}+h_{9} \frac{\partial \Phi_{p}}{\partial v_{z_{p}}}\right), \\
\tilde{\tau}_{\psi_{p}}=-\frac{\partial \Phi_{p}}{\partial v_{\psi_{p}}}, \tilde{\tau}_{\theta_{p}}=-\frac{\partial \Phi_{p}}{\partial v_{\theta_{p}}}, \tilde{\tau}_{\phi_{p}}=-\frac{\partial \Phi_{p}}{\partial v_{\phi_{p}}}
\end{gathered}
$$

We assume that the initial density of the five populations are Gaussian distributions $\rho_{0}^{p}, p=1,2,3,4,5$. And their centers in the spatial coordinates $(x, y, z)$ are located in $(-1,-5,-8),\left(4 \cos \left(\frac{2 \pi}{5}\right)-5,4 \sin \left(\frac{2 \pi}{5}\right)-5,-6\right),\left(4 \cos \left(\frac{4 \pi}{5}\right)-\right.$ $\left.5,4 \sin \left(\frac{4 \pi}{5}\right)-5,-6\right),\left(4 \cos \left(\frac{6 \pi}{5}\right)-5,4 \sin \left(\frac{6 \pi}{5}\right)-5,-4\right)$ and $\left(4 \cos \left(\frac{8 \pi}{5}\right)-5,4 \sin \left(\frac{8 \pi}{5}\right)-5,-2\right)$ with same standard deviation 0.5 , respectively. Noting that we set all other initial coordinates to zero (i.e. initial angular position, initial velocity, and initial angular velocity are all set to zero). We set the terminal cost to be a simple norm difference between the agents current position and the terminal distribution center position $(8,8,8)$, and we also want the agents to have zero velocity at terminal point, i.e.

$$
\begin{aligned}
& \mathcal{G}_{p}\left(\rho^{p}(\cdot, T), \rho^{-p}(\cdot, T)\right)=\int_{\mathbb{R}^{n}} \|\left(x_{p}, y_{p}, z_{p}, \psi_{p}, \ldots, v_{\phi_{p}}\right) \\
& -(8,8,8,0, \ldots, 0)\left\|_{2} \rho^{p}\left(\mathbf{x}^{p}, T\right) \mathrm{d} \mathbf{x}^{p}+\sum_{q \in V(p)} \int_{\mathbb{R}^{n}}\right\|\left(x_{q}, y_{q}, z_{q},\right. \\
& \left.\psi_{q}, \ldots, v_{\phi_{q}}\right)-(8,8,8,0, \ldots, 0) \|_{2} \rho^{q}\left(\mathbf{x}^{q}, T\right) \mathrm{d} \mathbf{x}^{q}
\end{aligned}
$$

In our experiments, we set the final time to be $T=4$.
We choose the interaction term to penalize congestion (a.k.a. interactive running cost) in our experiments, where the congestion is in the spatial positions, to encourage agents to spread out (and thus make mid-air collisions less likely). The first and second terms of the right formula of (28) are intragroup and inter-group congestion terms, respectively:

$$
\begin{aligned}
& \mathcal{F}_{p}\left(\rho^{p}\left(x_{p}, y_{p}, z_{p}\right), \rho^{-p}\left(x_{-p}, y_{-p}, z_{-p}\right)\right)=\gamma_{\text {cong }} \frac{1}{(2 \pi)^{\frac{3}{2}}} \int_{\mathbb{R}^{n}} \int_{\mathbb{R}^{n}} \\
& e^{\left(-\frac{1}{2}\left\|\left(x_{p}, y_{p}, z_{p}\right)-\left(\hat{x_{p}}, \hat{y_{p}}, \hat{z_{p}}\right)\right\|_{2}^{2}\right)} \mathrm{d} \rho^{p}\left(x_{p}, y_{p}, z_{p}\right) \mathrm{d} \rho^{p}\left(\hat{x_{p}}, \hat{y_{p}}, \hat{z_{p}}\right) \\
& +\beta_{\text {cong }} \frac{1}{(2 \pi)^{\frac{3}{2}}} \sum_{q \in V(p)} \int_{\mathbb{R}^{n}} \int_{\mathbb{R}^{n}} e^{\left(-\frac{1}{2}\left\|\left(x_{p}, y_{p}, z_{p}\right)-\left(x_{q}, y_{q}, z_{q}\right)\right\|_{2}^{2}\right)} \\
& \mathrm{d} \rho^{p}\left(x_{p}, y_{p}, z_{p}\right) \mathrm{d} \rho^{q}\left(x_{q}, y_{q}, z_{q}\right)
\end{aligned}
$$

where in our experiments we put intra-group congestion coefficient $\gamma_{\text {cong }}=20$, and inter-group congestion coefficient $\beta_{\text {cong }}=1 e+4$.

In all experiments, our neural networks have three hidden layers, with 100 hidden units per layer. We use a residual neural network (ResNet) for both networks, with a skip connection weight of 0.5 . For $\Phi_{\omega}^{p}$, we use the Tanh activation function, and for $\mathrm{G}_{\theta}^{p}$, we use the ReLU activation function. For training, we use ADAM with $\beta=(0.5,0.9)$, learning rate $4 e-4$ for $\Phi_{\omega}^{p}$, learning rate $1 e-4$ for $\mathrm{G}_{\theta}^{p}$, weight decay of $1 e-4$ for both networks, batch size $250, \lambda=1$ (the HJB penalty parameter), and $\eta=2.5 e-3$ (the neighbors penalty parameter) in Algorithm 1. In the following figures, time is represented by color. Specifically, blue denotes starting time, red denotes final time, and the intermediate colors denote intermediate times. As in standard machine learning methods, all the plots in Sec. IV are generated using validation data, i.e., data not used in training, to gauge generalizability of CA-Net.

Fig. 3 shows the trajectories of $N=1000$ quadcopters under multi-population MFG method. Through the observation and analysis of the obtained trajectories corresponding to eight 


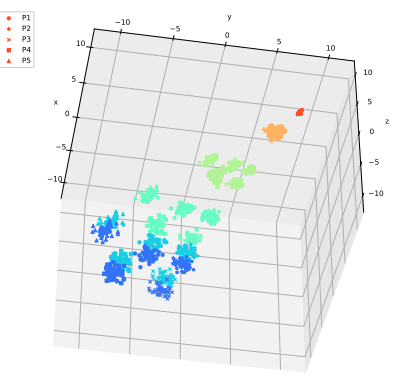

(a) no A, no B, no C

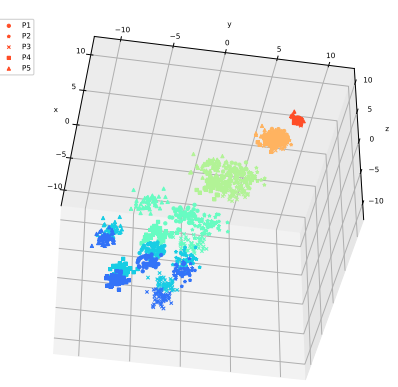

(e) with A, no B, no C

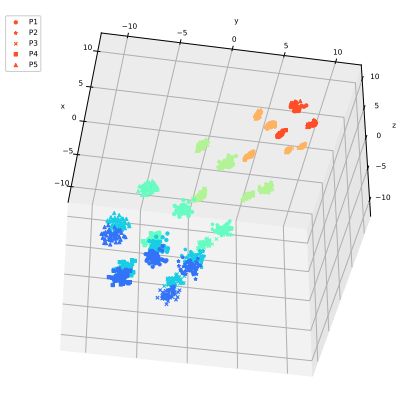

(b) no A, no B, with $\mathrm{C}$

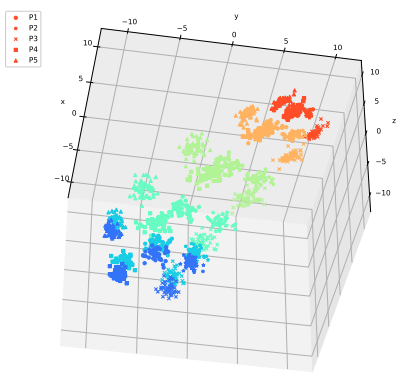

(f) with $\mathrm{A}$, no B, with $\mathrm{C}$

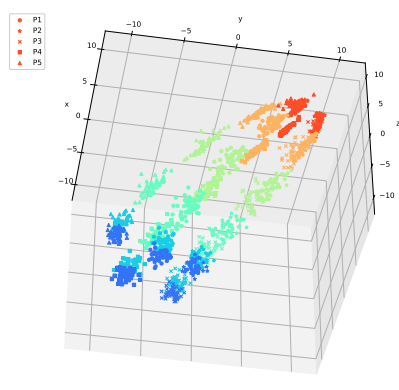

(c) no $\mathrm{A}$, with $\mathrm{B}$, with $\mathrm{C}$

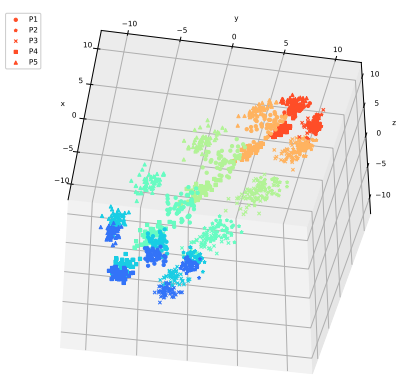

(g) with $\mathrm{A}$, with $\mathrm{B}$, with $\mathrm{C}$

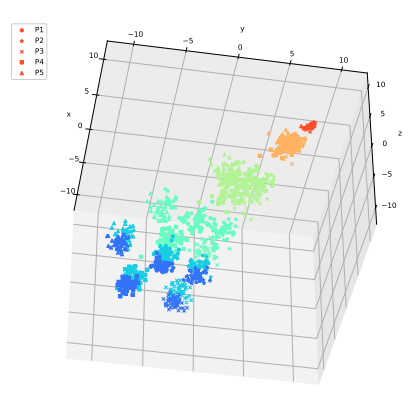

(d) no A, with B, no C

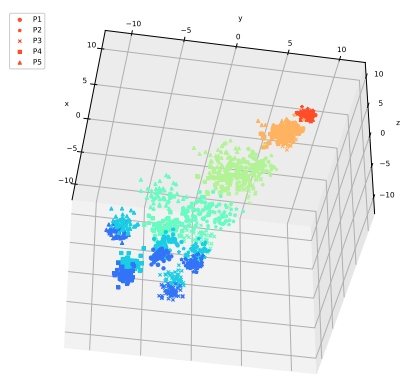

(h) with $\mathrm{A}$, with $\mathrm{B}$, no $\mathrm{C}$

Fig. 3: Illustration of multi-group quadcopters trajectory planning problem. The initial density (Bottom Left) and target density (Top Right) are Gaussians at the bottom and top, respectively, of these subplots domain. The five groups of agents start from the initial distribution, aiming to move towards the target distribution while avoiding intra-group and inter-group congestion. The colors in each subplot represent time, thus presenting the trajectories of the agents over time. Under different parameter configurations, the trajectories of the agents are different. A stands for noise $\sigma=1 e-2$, B for intra-group congestion coefficient $\gamma_{\text {cong }}=20$ and $\mathrm{C}$ for inter-group congestion coefficient $\beta_{\text {cong }}=1 e+4$. Comparing sub-pictures (a), (b), (c), (d), (e), (f), (g) and (h), it can be seen that with $\sigma$, the agents add an envelope of uncertainty; with $\gamma_{\text {cong }}$, the agents of intra-group spread out more as expected; with $\beta_{\text {cong }}$, the clusters are more diffuse.

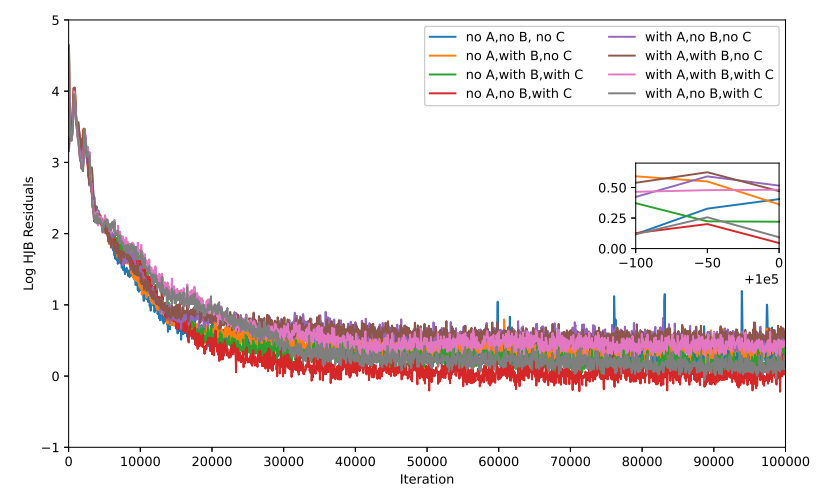

Fig. 4: Log HJB residual errors comparison with eight cases in Fig. 3. The horizontal axis is the number of iterations and the vertical axis is $\ell_{\mathrm{HJB}}$ in Algorithm 1.

groups of parameters, we can see that with noise $\sigma$, the agents add an envelope of uncertainty, so we do see the agents are not as streamlined as in the noiseless cases, and not getting as close to the terminal point of $(8,8,8)$ at terminal time. It is the comparison of subgraphs (e), (f), (g) and (h) with (a), (b), (c) and (d), respectively. Furthermore, in the presence of intragroup congestion coefficient $\gamma_{\text {cong }}$, the agents of intra-group spread out more as expected. It is the comparison of subgraphs between (d) and (a), (c) and (b), (h) and (e), (g) and (f). And in the presence of inter-group congestion coefficient $\beta_{\text {cong }}$, the clusters are more diffuse, and their terminal distributions do not fully gather to the expected terminal point of $(8,8,8)$. It is the comparison of subgraphs between (b) and (a), (c) and (d), (f) and (e), (g) and (h). We also plot the HJB residual errors in Fig. 4, i.e. $\ell_{\mathrm{HJB}}$ in Algorithm 1, which shows the convergence of CA-Net. Without an efficient strategy control, the HJB residuals under different cases are relatively high. HJB residuals drop fast after we apply a series of controls. After around $4 \mathrm{e}+4$ iterations, the error curves tend to be stable and close to zero, when we obtain an optimal control for quadcopters. Besides, it is obvious that the CA-Net algorithm converges faster without noise, and the red curve (no A, no B, with $\mathrm{C}$ )—without noise, without intra-group congestion, but with inter-group congestion-converges the fastest.

\section{B. Effect of Stochasticity Parameter $\sigma$}

We investigate the effect of the stochasticity parameter $\sigma$ on the behavior of the HJB residual errors and the multipopulation MFG solutions. 


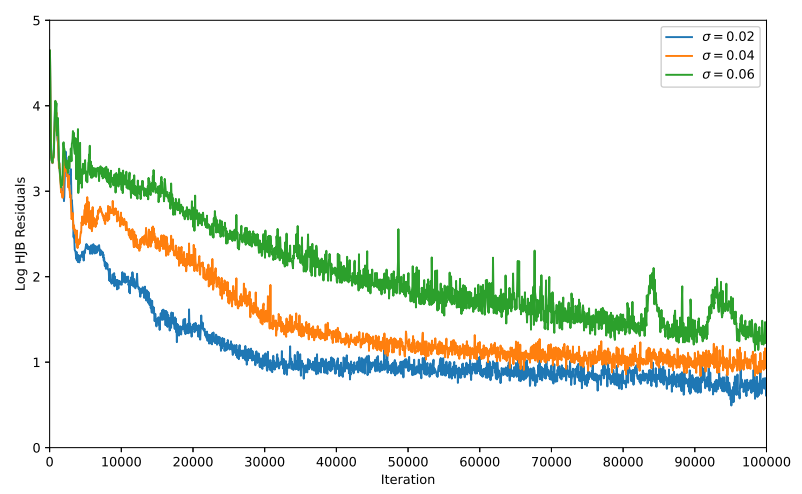

Fig. 5: Comparison of $\log$ HJB residual errors with stochasticity parameter $\sigma=0.02,0.04$ and 0.06 .
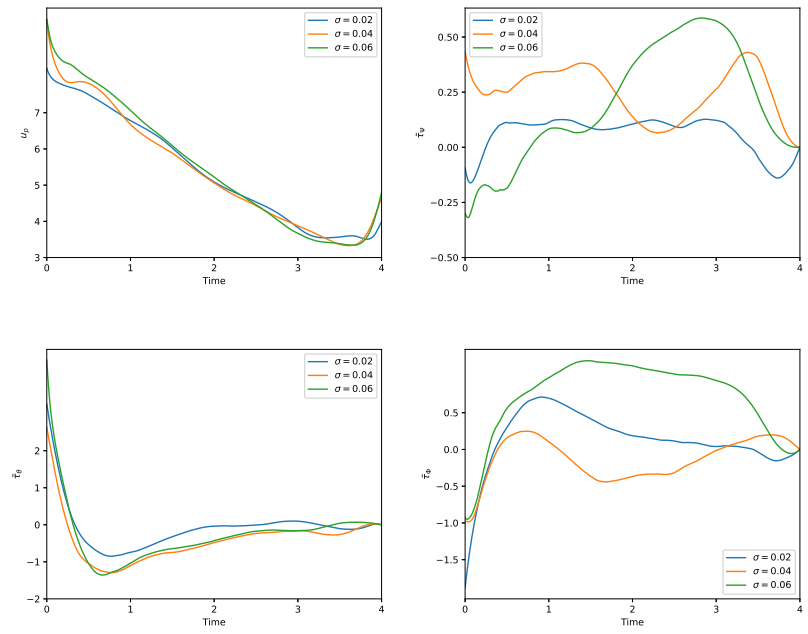

Fig. 6: Comparison of controls with different stochasticity parameters. The horizontal axis is time and the vertical axis is $u_{p}$ (Top Left), $\tilde{\tau}_{\psi_{p}}$ (Top Right), $\tilde{\tau}_{\theta_{p}}$ (Bottom Left), $\tilde{\tau}_{\phi_{p}}$ (Bottom Right), respectively.

In Fig. 5, we show the convergence behavior of the HJB residual errors of quadcopters with different stochasticity parameters. The HJB residual error decreases more and more slowly with the increase of noise $\sigma$. This means that the smaller $\sigma$, the faster the CA-Net algorithm converges.

In Fig. 6, we show the controls for multi-group quadcopters trajectory planning using $\sigma=0.02,0.04$, and 0.06. As $\sigma$ increases, the control curves of the agent fluctuates more.

In Fig. 7, we show the optimal velocity of quadcopters obtained by Algorithm 1. The initial average speed of drones is zero and it increases first and then decreases gradually when they approach the target distribution to minimize their loss functions. Moreover, before approaching the peak, when $\sigma$ is larger, the quadcopter tends to choose a faster speed. After the strategic speed change, at the terminal time $T=4$, the smaller $\sigma$ is, the closer the quadcopter terminal speed is to zero.

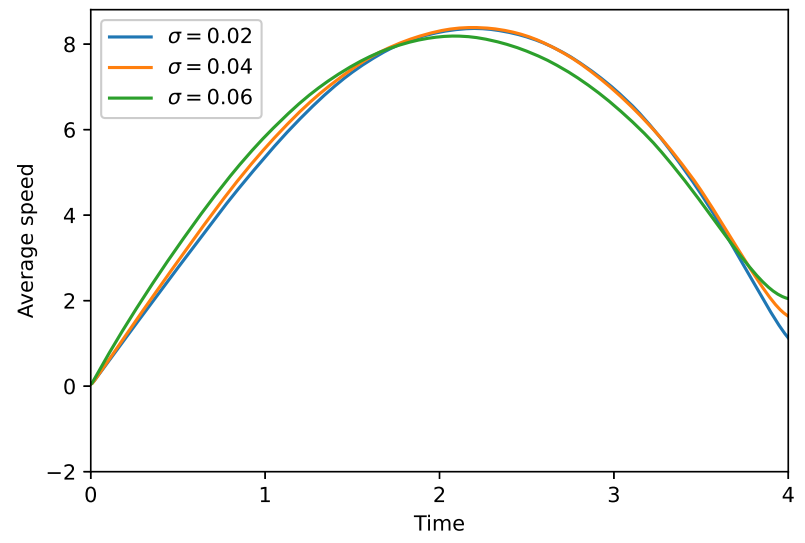

Fig. 7: Comparison of average speed with different stochasticity parameters.

\section{Comparison with Costs}

In order to further reveal the role of some important parameters in the design of CA-Net algorithm. We show the transport $\mathcal{L}_{p}$, intra-running $\mathcal{F}_{p}{ }^{\text {intra }}$, inter-running $\mathcal{F}_{p}{ }^{\text {inter }}$, terminal $\mathcal{G}_{p}$ and objective $J_{p}$ costs in Table I under the change of parameters.

From Table I, first, compare the influence of the $\sigma$ parameter and fix other parameters. From the first line to the third line, it can be seen that the larger stochasticity $\sigma$, the greater the transport cost $\mathcal{L}_{p}$, the smaller the intragroup collision cost $\mathcal{F}_{p}{ }^{\text {intra }}$, and the greater the total cost $J_{p}$. Comparing lines 6 and 10, 7 and 11, 8 and 12, 9 and 13, we can see that if $\sigma$ exists, the total cost $J_{p}$ is relatively large. Second, comparing the influence of parameter $\eta$, it can be seen from the fourth and fifth lines that if the neighbor dual penalty item exists, the smaller the transport cost $\mathcal{L}_{p}$, the greater the intragroup $\mathcal{F}_{p}$ intra and intergroup $\mathcal{F}_{p}{ }^{\text {inter }}$ cost, the constant terminal cost $\mathcal{G}_{p}$, and the smaller the total cost $J_{p}$. Third, compare the influence of the parameter $\lambda$. It can be seen from lines 13 and 14 that if the HJB penalty item exists, the smaller the transport cost $\mathcal{L}_{p}$, the smaller the total cost $J_{p}$. Fourth, compare the influence of

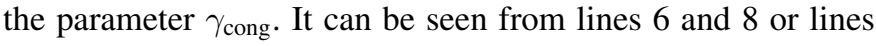
10 and 12 that if the intra-group congestion coefficient $\gamma_{\text {cong }}$ exists, the intragroup collision cost $\mathcal{F}_{p}{ }^{\text {intra }}$ will be relatively small. Fifth, compare the impact of parameter $\beta_{\text {cong. It can }}$ be seen from lines 6 and 7 or lines 10 and 11 that if the inter-group congestion coefficient $\beta_{\text {cong }}$ exists, the intergroup collision cost $\mathcal{F}_{p}$ inter is relatively small. Sixth, compare the influence of the network parameter Width, i.e. the network width of each layer. By observing lines 13 and 15, we can see that smaller networks can also achieve convergence, and the total cost $J_{p}$ is small. As shown in Fig. 8, for networks with the same depth, the training convergence speed is faster if each layer is appropriately widened. The larger networks have good robustness and better generalization in complex cases. Finally, comparing the parameter Time Steps, that is, lines 13 and 16, the Time Steps is reduced from 600 to 300, and the total cost fluctuates by nearly $1 \%$, which means that our network is convergent and stable. 
TABLE I: Comparison with Costs

\begin{tabular}{ccccccccccccc}
\hline \hline Number & Width & Time Steps & $\sigma$ & $\eta$ & $\lambda$ & $\gamma_{\text {cong }}$ & $\beta_{\text {cong }}$ & $\mathcal{L}_{p}$ & $\mathcal{F}_{p}{ }^{\text {intra }}$ & $\mathcal{F}_{p}$ inter & $\mathcal{G}_{p}$ & $J_{p}$ \\
\hline 1 & 100 & 600 & 0.02 & $2.50 \mathrm{e}-03$ & 1 & 20 & $1.00 \mathrm{e}+05$ & $1.68 \mathrm{e}+01$ & $2.33 \mathrm{e}-02$ & $5.03 \mathrm{e}-02$ & $1.45 \mathrm{e}+01$ & $3.14 \mathrm{e}+01$ \\
2 & 100 & 600 & 0.04 & $2.50 \mathrm{e}-03$ & 1 & 20 & $1.00 \mathrm{e}+05$ & $1.79 \mathrm{e}+01$ & $1.85 \mathrm{e}-02$ & $5.54 \mathrm{e}-02$ & $1.49 \mathrm{e}+01$ & $3.29 \mathrm{e}+01$ \\
3 & 100 & 600 & 0.06 & $2.50 \mathrm{e}-03$ & 1 & 20 & $1.00 \mathrm{e}+05$ & $1.99 \mathrm{e}+01$ & $1.26 \mathrm{e}-02$ & $3.15 \mathrm{e}-02$ & $1.47 \mathrm{e}+01$ & $3.47 \mathrm{e}+01$ \\
4 & 100 & 600 & 0.01 & 0 & 1 & 20 & $1.00 \mathrm{e}+05$ & $1.64 \mathrm{e}+01$ & $2.33 \mathrm{e}-02$ & $4.30 \mathrm{e}-02$ & $1.46 \mathrm{e}+01$ & $3.11 \mathrm{e}+01$ \\
5 & 100 & 600 & 0.01 & $1.00 \mathrm{e}-02$ & 1 & 20 & $1.00 \mathrm{e}+05$ & $1.52 \mathrm{e}+01$ & $2.86 \mathrm{e}-02$ & $5.22 \mathrm{e}-02$ & $1.46 \mathrm{e}+01$ & $2.98 \mathrm{e}+01$ \\
6 & 100 & 600 & 0 & $2.50 \mathrm{e}-03$ & 1 & 0 & 0 & $1.56 \mathrm{e}+01$ & $4.73 \mathrm{e}-02$ & $1.41 \mathrm{e}-01$ & $1.41 \mathrm{e}+01$ & $2.98 \mathrm{e}+01$ \\
7 & 100 & 600 & 0 & $2.50 \mathrm{e}-03$ & 1 & 0 & $1.00 \mathrm{e}+05$ & $1.57 \mathrm{e}+01$ & $4.71 \mathrm{e}-02$ & $6.85 \mathrm{e}-02$ & $1.45 \mathrm{e}+01$ & $3.03 \mathrm{e}+01$ \\
8 & 100 & 600 & 0 & $2.50 \mathrm{e}-03$ & 1 & 20 & 0 & $1.65 \mathrm{e}+01$ & $3.11 \mathrm{e}-02$ & $5.64 \mathrm{e}-02$ & $1.41 \mathrm{e}+01$ & $3.07 \mathrm{e}+01$ \\
9 & 100 & 600 & 0 & $2.50 \mathrm{e}-03$ & 1 & 20 & $1.00 \mathrm{e}+05$ & $1.55 \mathrm{e}+01$ & $2.86 \mathrm{e}-02$ & $6.08 \mathrm{e}-02$ & $1.47 \mathrm{e}+01$ & $3.03 \mathrm{e}+01$ \\
10 & 100 & 600 & 0.01 & $2.50 \mathrm{e}-03$ & 1 & 0 & 0 & $1.59 \mathrm{e}+01$ & $3.76 \mathrm{e}-02$ & $9.28 \mathrm{e}-02$ & $1.40 \mathrm{e}+01$ & $3.01 \mathrm{e}+01$ \\
11 & 100 & 600 & 0.01 & $2.50 \mathrm{e}-03$ & 1 & 0 & $1.00 \mathrm{e}+05$ & $1.61 \mathrm{e}+01$ & $3.30 \mathrm{e}-02$ & $5.83 \mathrm{e}-02$ & $1.49 \mathrm{e}+01$ & $3.11 \mathrm{e}+01$ \\
12 & 100 & 600 & 0.01 & $2.50 \mathrm{e}-03$ & 1 & 20 & 0 & $1.66 \mathrm{e}+01$ & $2.49 \mathrm{e}-02$ & $5.40 \mathrm{e}-02$ & $1.42 \mathrm{e}+01$ & $3.08 \mathrm{e}+01$ \\
13 & 100 & 600 & 0.01 & $2.50 \mathrm{e}-03$ & 1 & 20 & $1.00 \mathrm{e}+05$ & $1.65 \mathrm{e}+01$ & $2.43 \mathrm{e}-02$ & $6.27 \mathrm{e}-02$ & $1.47 \mathrm{e}+01$ & $3.13 \mathrm{e}+01$ \\
14 & 100 & 600 & 0.01 & $2.50 \mathrm{e}-03$ & 0 & 20 & $1.00 \mathrm{e}+05$ & $1.68 \mathrm{e}+01$ & $2.76 \mathrm{e}-02$ & $5.89 \mathrm{e}-02$ & $1.46 \mathrm{e}+01$ & $3.15 \mathrm{e}+01$ \\
15 & 50 & 600 & 0.01 & $2.50 \mathrm{e}-03$ & 1 & 20 & $1.00 \mathrm{e}+05$ & $1.53 \mathrm{e}+01$ & $2.27 \mathrm{e}-02$ & $6.45 \mathrm{e}-02$ & $1.51 \mathrm{e}+01$ & $3.04 \mathrm{e}+01$ \\
16 & 100 & 300 & 0.01 & $2.50 \mathrm{e}-03$ & 1 & 20 & $1.00 \mathrm{e}+05$ & $1.63 \mathrm{e}+01$ & $2.57 \mathrm{e}-02$ & $5.97 \mathrm{e}-02$ & $1.45 \mathrm{e}+01$ & $3.09 \mathrm{e}+01$ \\
\hline \hline
\end{tabular}

All values were approximated using the validation points; $\sigma$, stochasticity; $\eta$, neighbor penalty coefficient; $\lambda$, HJB penalty coefficient; $\gamma_{\text {cong }}$, intra-group congestion coefficient; $\beta_{\text {cong }}$, inter-group congestion coefficient; $\mathcal{L}_{p}$, transport costs; $\mathcal{F}_{p}$ intra , intra-running costs; $\mathcal{F}_{p}{ }^{\text {inter }}$, inter-running costs; $\mathcal{G}_{p}$, terminal costs; $J_{p}$, objective costs.

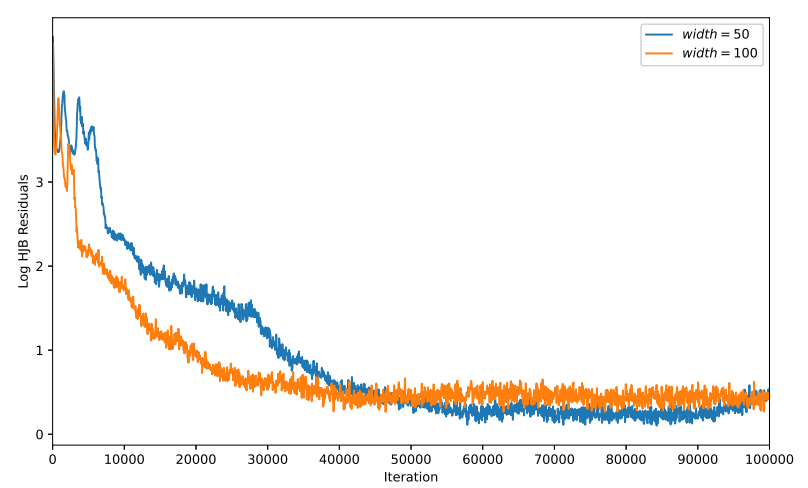

Fig. 8: Comparison of log HJB residual errors under different network widths.

\section{Comparison with Baselines}

As the last part of the numerical experiment, we verify the superiority of our method by comparing it to some typical baseline methods, including cluster game [77], HJB-NN [65], Lax-Friedrichs [62], ML [31], APAC-Net [56]. For more details of these baseline methods, please refer to the related documents [31], [56], [62], [65], [77], etc. Below we give the performance comparison of the above algorithms in Table II.

For cluster game [77], it is a game model for small-scale multi-group agent control problems, and that communicates through a given point-to-point communication topology. The equilibrium calculation adopts an analytical solution based on grid calculation. The solution space is generally lowdimensional, and special interference is usually not considered.
HJB-NN [65] is a neural network approach for solving highdimensional optimal control problems. This method can be used for large-scale group modeling. And the information interaction between agents is point-to-point communication, which means that the communication cost is relatively high. Literature [62] describes a multi-population social network MFG problem, which is solved analytically by an adjoint method. Its corresponding algorithm uses a numerical scheme called the Lax-Friedrichs scheme [64] belonging to the finite difference method. Therefore, the numerical solution is cursed by dimension and is only suitable for low-dimensional solution space. Literature [31] provides a machine learning framework for solving high-dimensional MFG and mean-field control problems. However, it is limited to solving the single group deterministic MFGs. APAC-Net [56] is an alternating population and agent control neural network for solving highdimensional stochastic MFGs. It is indeed suitable for largescale homogeneous agent systems. Our proposed CA-Net is a nonlinear coupled alternating neural network tailored for multi-population high-dimensional stochastic MFGs, which can be induced by optimal control problems of large-scale multi-agent heterogeneous systems. To sum up, we can see the superiority of the CA-Net algorithm in all-around performance from Table II.

\section{CONClusion}

We formally propose a numerical solution scheme for the multi-population high-dimensional stochastic MFG system. The execution algorithm here is CA-Net, a nonlinear coupled alternating neural network. To this end, the proposed modeling framework strictly represents the large-scale heterogeneous multi-agent game system as a multi-population MFG model. 
TABLE II: Comparison with baseline methods

\begin{tabular}{ccccccc}
\hline \hline Method & Communication & Computation & Scale $(N)$ & Dimension $(n)$ & Population $(P)$ & Stochasticity $(\sigma)$ \\
\hline Cluster game [77] & $\mathcal{O}(N)$ & $\mathcal{O}(n !)$ & Small & Low & Multiple & No \\
HJB-NN [65] & $\mathcal{O}(N)$ & $\mathcal{O}(n)$ & Large & High & Single & No \\
Lax-Friedrichs [62] & $\mathcal{O}(1)$ & $\mathcal{O}(n !)$ & Large & Low & Multiple & Yes \\
ML [31] & $\mathcal{O}(1)$ & $\mathcal{O}(n)$ & Large & High & Single & No \\
APAC-Net [56] & $\mathcal{O}(1)$ & $\mathcal{O}(n)$ & Large & High & Single & Yes \\
CA-Net & $\mathcal{O}(1)$ & $\mathcal{O}(n)$ & Large & High & Multiple & Yes \\
\hline \hline
\end{tabular}

$\mathcal{O}()$, infinitesimal of the same order.

Our algorithm avoids the use of spatial grids by parameterizing the value functions and the density functions of multiple populations by two sets of neural networks, respectively. We design a multiple generators and multiple discriminators GAN, where density functions act as generative networks and value functions act as discriminative networks. Unlike other GANs, CA-Net incorporates the structure of multi-population MFGs, which absolves the network from learning an entire multi-population MFG solution from the ground up-provides numerical solutions for the multi-population MFG and improves the solution efficiency. Consequently, our method is geared toward multi-population high-dimensional instances of MFGs that are beyond reach with existing solution methods. CA-Net, therefore, sets the stage for solving realistic multipopulation high-dimensional MFGs arising in, e.g., crowd motion, swarm robotics, epidemic modeling. To verify the feasibility of the multi-population MFG model, we provide a practical numerical simulation case, multi-group quadcopters trajectory planning. The convergence results about HJB residual error, control, average speed show the effectiveness of the CA-Net algorithm. To reveal the role of multi-dimensional parameters in the design of the CA-Net, we show the costs of transport, intra-running, inter-running, terminal, and objective under the change of these parameters. Finally, the comparison with baseline methods-cluster game, HJB-NN, Lax-Friedrichs, ML, APAC-Net-makes clear the advanced nature of our solution method. In the future, we will consider the application and adaptation of the CA-Net method in more complex scenarios of heterogeneous multi-agent systems, such as complex communication conditions (time-varying, partial rejection) and obstacles (static, dynamic).

\section{ACKNOWLEDGMENT}

We thank prof. Zhiming Zheng for his fruitful discussions, valuable opinions, and guidance. This work was supported by the Science and Technology Innovation 2030-Key Project under Grant 2020AAA0108200.

\section{REFERENCES}

[1] J. M. Lasry, P. L. Lions and Jeux à champ moyen, I. Le cas stationnaire, C. R. Math. Acad. Sci. Paris 343, pp. 619-625, 2006.

[2] J. M. Lasry, P. L. Lions and Jeux à champ moyen, II. Horizon fini et contrôle optimal, C. R. Math. Acad. Sci. Paris 343, pp. 679-684, 2006.

[3] J. M. Lasry and P. L. Lions, Mean field games, Japanese Journal of Mathematics, vol. 2, no. 1, pp. 229-260, 2007.
[4] M. Y. Huang, P. E. Caines and R. P. Malhamé, Individual and mass behaviour in large population stochastic wireless power control problems: centralized and nash equilibrium solutions, in: IEEE Conference on Decision and Control, HI, USA, December, pp. 98-103, 2003.

[5] M. Y. Huang, R. P. Malhamé and P. E. Caines, Large population stochastic dynamic games: Closed-loop McKean-Vlasov systems and the Nash certainty equivalence principle, Commun. Inf. Syst. 6, pp. 221-251, 2006.

[6] M. Y. Huang, P. E. Caines and R. P. Malhamé, Large-population costcoupled LQG problems with nonuniform agents: Individual-mass behavior and decentralized $\epsilon$-Nash equilibria, IEEE Trans. Autom. Control 52, pp. 1560-1571, 2007.

[7] Z. Zhou and H. Xu, A novel mean-field-game-type optimal control for very large-scale multiagent systems, IEEE Transactions on Cybernetics, 2020.

[8] O. Guéant, J. M. Lasry and P. L. Lions, Mean field games and applications, in ParisPrinceton Lectures on Mathematical Finance 2010, R. Carmona et al., Eds. (Lecture Notes in Mathematics, Springer, Berlin, 2011), vol. 2003, pp. 205-266, 2010.

[9] Y. Achdou, F. J. Buera, J. M. Lasry, P. L. Lions and B. Moll, Partial differential equation models in macroeconomics, Philos. Trans. R. Soc. A 372, 20130397, 2014.

[10] D. A. Gomes, L. Nurbekyan and E. A. Pimentel, Economic Models and Mean-Field Games Theory, Instituto Nacional de Matemática Pura e Aplicada, Rio de Janeiro, Brazil, 2015.

[11] Y. Achdou, J. Han, J. M. Lasry, P. L. Lions and B. Moll, Income and wealth distribution in macroeconomics: A continuous-time approach, Working pap. 23732, National Bureau of Economic Research, 2017.

[12] A. Lachapelle, J. M. Lasry, C. A. Lehalle and P. L. Lions, Efficiency of the price formation process in presence of high frequency participants: A mean field game analysis, Math. Financ. Econ. 10, pp. 223-262, 2016.

[13] D. Firoozi and P. E. Caines, An optimal execution problem in finance targeting the market trading speed: An MFG formulation, in 2017 IEEE 56th Annual Conference on Decision and Control (CDC) (Institute of Electrical and Electronics Engineers, 2017), pp. 7-14, 2017.

[14] P. Cardaliaguet and C. A. Lehalle, Mean field game of controls and an application to trade crowding, Math. Financ. Econ. 12, pp. 335-363, 2018.

[15] P. Casgrain, S. Jaimungal, Algorithmic trading in competitive markets with mean field games, SIAM News 52, pp. 1-2, 2019.

[16] D. A. Gomes and J. Saúde, A mean-field game approach to price formation in electricity markets, arXiv:1807.07088 (19 November 2018).

[17] A. C. Kizilkale, R. Salhab and R. P. Malhamé, An integral control formulation of mean field game based large scale coordination of loads in smart grids, Automatica 100, pp. 312-322, 2019.

[18] A. De Paola, V. Trovato, D. Angeli and G. Strbac, A mean field game approach for distributed control of thermostatic loads acting in simultaneous energy-frequency response markets, IEEE Transactions Smart Grid 10, pp. 5987-5999, 2019.

[19] A. Lachapelle and M. T. Wolfram, On a mean field game approach modeling congestion and aversion in pedestrian crowds, Transp. Res. Part B Methodol. 45, pp. 1572-1589, 2011.

[20] M. Burger, M. Di Francesco, P. A. Markowich and M. T. Wolfram, Mean field games with nonlinear mobilities in pedestrian dynamics, Discrete Contin. Dyn. Syst. Ser. B 19, pp. 1311-1333, 2014.

[21] A. Aurell and B. Djehiche, Mean-field type modeling of nonlocal crowd aversion in pedestrian crowd dynamics, SIAM J. Contr. Optim. 56, pp. 434-455, 2018.

[22] Y. Achdou and J. M. Lasry, Mean field games for modeling crowd motion, in Contributions to Partial Differential Equations and Applica- 
tions, B. Chetverushkin et al., Eds. (Computational Methods in Applied Sciences, Springer, Cham, Switzerland, 2019), vol. 47, pp. 17-42, 2019.

[23] Z. Liu, B. Wu and $\mathrm{H}$. Lin, A mean field game approach to swarming robots control, In 2018 Annual American Control Conference (ACC), IEEE, pp. 4293-4298, 2018

[24] K. Elamvazhuthi and S. Berman, Mean-field models in swarm robotics: a survey, Bioinspiration \& Biomimetics, vol. 15, no. 1, 2019.

[25] W. Lee, S. Liu, H. Tembine and S. Osher, Controlling propagation of epidemics via mean-field games, UCLA CAM preprint:20-19, 2020.

[26] S. L. Chang, M. Piraveenan, P. Pattison and M. Prokopenko, Game theoretic modelling of infectious disease dynamics and intervention methods: a review, Journal of Biological Dynamics, vol. 14, no. 1, pp. $57-89,2020$.

[27] E. Weinan, J. Han and Q. Li, A mean-field optimal control formulation of deep learning, Research in the Mathematical Sciences, vol. 6, no. 1, 2019.

[28] X. Guo, A. Hu, R. Xu and J. Zhang, Learning mean-field games, In Advances in Neural Information Processing Systems, pp. 4967-4977, 2019.

[29] R. Carmona, M. Laurière and Z. Tan, Linear-quadratic mean-field reinforcement learning: convergence of policy gradient methods, arXiv: $1910.04295,2019$.

[30] P. M. Welch, K. Ø. Rasmussen and C. F. Welch, Describing nonequilibrium soft matter with mean field game theory, J. Chem. Phys. 150, 174905, 2019.

[31] L. Ruthotto, S. J. Osher, W. Li, L. Nurbekyan and S. W. Fung, A machine learning framework for solving high-dimensional mean field game and mean field control problems, Proceedings of the National Academy of Sciences, vol. 117, no. 17, pp. 9183-9193, 2020.

[32] R. Bellman, Dynamic programming, Princeton University Press, Princeton, NJ, 1957.

[33] J. Han, J. Arnulf and E. Weinan, Solving high-dimensional partial differential equations using deep learning, Proceedings of the National Academy of Sciences, vol. 115, no. 34, pp. 8505-8510, 2018.

[34] M. Fujii, Probabilistic Approach to Mean Field Games and Mean Field Type Control Problems with Multiple Populations, arXiv: 1911. 11501v3 [math. PR] 2 Nov 2020, 2020.

[35] Y. Achdou and V. Perez, Iterative strategies for solving linearized discrete mean field games systems, Netw Heterog Media, vol. 7, no. 2, pp. 197-217, 2012.

[36] Y. Achdou, F. Camilli and I. Capuzzo-Dolcetta, Mean field games: numerical methods for the planning problem, SIAM J Control Optim, vol. 50, no. 1, pp. 77-109, 2012

[37] O. Guéant, Mean field games equations with quadratic Hamiltonian: a specific approach, Math Models Methods Appl Sci, vol. 22, no. 9, 2012.

[38] O. Guéant, Mean field games with a quadratic Hamiltonian: a constructive scheme. In: Advances in dynamic games, Ann Int Soc Dyn Games. Birkhäuser/Springer, New York, vol. 12, pp. 229-241, 2012.

[39] O. Guéant, New numerical methods for mean field games with quadratic costs, Netw Heterog Media, vol. 7, no. 2, pp. 315-336, 2012.

[40] F. Camilli, F. Silva A semi-discrete approximation for a first order mean field game problem, Netw Heterog Media, vol. 7, no. 2, pp. 263-277, 2012.

[41] F. Camilli, A. Festa, D. Schieborn An approximation scheme for a HamiltonJacobi equation defined on a network, Appl Numer Math, vol. 73, pp. 33-47, 2013.

[42] Y. Achdou, Finite difference methods for mean field games. In: Hamilton-Jacobi equations: approximations, numerical analysis and applications, Lecture notes in mathematics,Springer, Heidelberg, vol. 2074, pp. 147, 2013.

[43] E. Carlini, F. J. Silva A fully discrete semi-Lagrangian scheme for a first order mean field game problem, SIAM J Numer Anal, vol. 52, no. 1, pp. 45-67, 2014.

[44] Y. Achdou and A. Porretta, Convergence of a finite difference scheme to weak solutions of the system of partial differential equations arising in mean field games, SIAM J Numer Anal, vol. 54, no. 1, pp. 161-186, 2016.

[45] S. Cacace and F. Camilli, Ergodic problems for Hamilton-Jacobi equations: yet another but efficient numerical method, preprint, 2016.

[46] N. Almulla, R. Ferreira and D. Gomes, Two numerical approaches to stationary mean-field games, Dyn. Games Appl., vol. 7, no. 4, pp. 657682, 2017.

[47] P. Cardaliaguet and S. Hadikhanloo, Learning in mean field games: The fictitious play, ESAIM Control Optim. Calculus Variations, vol. 23, no. 2, pp. 569-591, 2017

[48] D. Gomes R. M. Velho and M. T. Wolfram, Dual two-state mean-field games, In: Proceedings CDC, 2014
[49] D. Gomes R. M. Velho and M. T. Wolfram, Socio-economic applications of finite state mean field games, Philos Trans R Soc Lond Ser A Math Phys Eng Sci, vol.372, no.2028, 2014.

[50] Y. Achdou and I. Capuzzo-Dolcetta, Mean field games: numerical methods, SIAM J Numer Anal, vol. 48, no. 3, pp. 1136-1162, 2010.

[51] J. D. Benamou, G. Carlier and F. Santambrogio, Variational mean field games, In Active Particles, vol. 1, pp. 141-171, Springer International Publishing, 2017.

[52] Y. T. Chow, W. Li, S. Osher and W. Yin, Algorithm for hamiltonjacobi equations in density space via a generalized hopf formula, Journal of Scientific Computing, vol. 80, no. 2, pp. 1195-1239, 2019.

[53] Y. T. Chow, J. Darbon, S. Osher and W. Yin, Algorithm for overcoming the curse of dimensionality for time-dependent non-convex hamiltonjacobi equations arising from optimal control and differential games problems, Journal of Scientific Computing, vol. 73, no. 2, pp. 617-643, 2017.

[54] Y. T. Chow, J. Darbon, S. Osher and W. Yin, Algorithm for overcoming the curse of dimensionality for certain non-convex hamiltonjacobi equations, projections and differential games, Annals of Mathematical Sciences and Applications, vol. 3, no. 2, pp. 369-403, 2018.

[55] M. Jacobs, F. Léger, W. Li and S. Osher, Solving large-scale optimization problems with a convergence rate independent of grid size. SIAM Journal on Numerical Analysis, SIAM Journal on Numerical Analysis, vol. 57, no. 3, pp. 1100-1123, 2019

[56] A. T. Lin, S. W. Fung, W. Li, L. Nurbekyan and S. J. Osher, Alternating the population and control neural networks to solve high-dimensional stochastic mean-field games, Proceedings of the National Academy of Sciences, vol. 118, no. 31, pp. 0027-8424, 2021.

[57] I. Goodfellow, J. Pouget-Abadie, M. Mirza, B. Xu, D. Warde-Farley, S. Ozair, A. Courville and Y. Bengio, Generative adversarial nets, In Advances in neural information processing systems, pp. 2672-2680, 2014

[58] E. Feleqi, The derivation of ergodic mean field game equations for several population of players, Dynamic Games and Applications, vol. 3, pp. 523-536, 2013.

[59] M. Cirant, Multi-population mean field games system with Neumann boundary conditions, J. Math. Pures Appl., vol. 103, pp. 1294-1315, 2015.

[60] Y. Achdou, M. Bardi and M. Cirant, Mean field games models of segregation, Mathematical Models and Methods in Applied Sciences, vol. 27, no. 1, pp. 75-113, 2017.

[61] Lachapelle and M. Wolfram, On a mean field game approach modeling congestion and aversion in pedestrian crowds, Transportation Research Part B, 45, pp. 1572-1589, 2011.

[62] R. A. Banez, H. Gao, L. Li et al., Belief and opinion evolution in social networks based on a multi-population mean field game approach, ICC 2020-2020 IEEE International Conference on Communications (ICC). IEEE, 2020.

[63] A. Bensoussan, H. Tao and M. Laurière, Mean field control and mean field game models with several populations, Minimax Theory and its Applilications, 03, No. 2, pp. 173-209, 2018.

[64] J. M. Schulte, Adjoint methods for Hamilton-Jacobi-Bellman equations, Diploma Thesis, University of Munster, Germany, Nov. 2010.

[65] D. Onken and L. Nurbekyan and X. Li et al., A Neural Network Approach for High-Dimensional Optimal Control, arXiv: 2104. 03270v1 [math.OC] 7 Apr 2021, 2021.

[66] Y. W. Wang and Y. Lei, T. Bian and Z. H. Guan Distributed control of nonlinear multiagent systems with unknown and nonidentical control directions via event-triggered communication, IEEE Trans. Cybern., vol. 50, no. 5, pp. 1820-1832, May 2020.

[67] R. Olfati-Saber, J. A. Fax and R. M. Murray, Consensus and cooperation in networked multi-agent systems, Proc. IEEE, vol. 95, no. 1, pp. 215-233, Jan. 2007.

[68] H. Gao, W. Lee and W. Li, et al., Energy-efficient velocity control for massive numbers of rotary-wing UAVs: a mean field game approach, GLOBECOM 2020-2020 IEEE Global Communications Conference. IEEE, 2020.

[69] P. Cardaliaguet and P. J. Graber, Mean field games systems of first order, ESAIM: Control, Optimisation and Calculus of Variations, vol. 21, no. 3, pp. 690-722, 2015.

[70] P. Cardaliaguet, P. J. Graber, A. Porretta and D. Tonon, Second order mean field games with degenerate diffusion and local coupling, Nonlinear Differential Equations and Applications NoDEA, vol. 22, no. 5, pp. 1287 1317, 2015.

[71] M. Cirant and L. Nurbekyan, The variational structure and time-periodic solutions for mean-field games systems, Minimax Theory Appl., vol. 3, no. 2, pp. 227-260, 2018. 
[72] D. Onken, S. W. Fung, X. Li and L. Ruthotto, OT-Flow: Fast and accurate continuous normalizing flows via optimal transport, arXiv: 2006. 00104, 2020.

[73] O. Guéant, J. M. Lasry and P. L. Lions, Mean field games and applications, In Paris-Princeton lectures on mathematical finance 2010, pp. 205-266, Springer, 2011.

[74] A. T. Lin, Y. T. Chow and S. J. Osher, A splitting method for overcoming the curse of dimensionality in HamiltonJacobi equations arising from nonlinear optimal control and differential games with applications to trajectory generation, Communications in Mathematical Sciences, vol. 16, no. 7, pp. 1933-1973, 2018.

[75] D. Onken, L. Nurbekyan, X. Li, S. W. Fung, S. Osher and L. Ruthotto, $A$ Neural Network Approach for High-Dimensional Optimal Control, arXiv: 2104.03270, 2021.

[76] L. R. G. Carrillo, A. E. D. López, R. Lozano and C. Pégard, Modeling the quad-rotor mini-rotorcraft, In Quad Rotorcraft Control, Springer, pp. 23-34, 2013.

[77] M. Ye, G. Hu, R. Lozano and F. L. Lewis et al., A unified strategy for solution seeking in graphical n-coalition noncooperative games, IEEE Transactions on Automatic Control, vol. 64, no. 11, pp. 4645-4652, 2019. 